\title{
The Vertical Structure of the Surface Wave Radiation Stress for Circulation over a Sloping Bottom as Given by Thickness-Weighted-Mean Theory
}

\author{
HIDENORI AIKI \\ Research Institute for Global Change, Japan Agency for Marine-Earth Science and Technology, Yokohama, Japan \\ RICHARD J. GREATBATCH \\ GEOMAR|Helmholtz-Zentrum für Ozeanforschung Kiel, Kiel, Germany
}

(Manuscript received 23 March 2012, in final form 2 July 2012)

\begin{abstract}
Previous attempts to derive the depth-dependent expression of the radiation stress have led to a debate concerning (i) the applicability of the Mellor approach to a sloping bottom, (ii) the introduction of the delta function at the mean sea surface in the later papers by Mellor, and (iii) a wave-induced pressure term derived in several recent studies. The authors use an equation system in vertically Lagrangian and horizontally Eulerian (VL) coordinates suitable for a concise treatment of the surface boundary and obtain an expression for the depth-dependent radiation stress that is consistent with the vertically integrated expression given by Longuet-Higgins and Stewart. Concerning (i)-(iii) above, the difficulty of handling a sloping bottom disappears when wave-averaged momentum equations in the VL coordinates are written for the development of (not the Lagrangian mean velocity but) the Eulerian mean velocity. There is also no delta function at the sea surface in the expression for the depth-dependent radiation stress. The connection between the wave-induced pressure term in the recent studies and the depth-dependent radiation stress term is easily shown by rewriting the pressure-based form stress term in the thickness-weighted-mean momentum equations as a velocity-based term that contains the time derivative of the pseudomomentum in the VL framework.
\end{abstract}

\section{Introduction}

The radiation stress term of Longuet-Higgins and Stewart (1964, hereafter LHS64) and Mellor $(2003,2005)$ can be regarded as the sum of the (horizontal) Reynolds stress term and the negative of the form stress term. The former represents the residual effect of momentum advection, and the latter represents the residual effect of pressure perturbations. Both terms are clear in the depth-integrated framework of LHS64 and have long been used in the community to describe the residual effect of surface waves on circulation in the upper ocean. However there is confusion concerning the analytical expression for the depth-dependent form stress term (while the analytical expression of the depth-dependent Reynolds stress term is clear). The depth-dependent

Corresponding author address: Hidenori Aiki, Research Institute for Global Change, Japan Agency for Marine-Earth Science and Technology, Yokohama, Japan.

E-mail: aiki@jamstec.go.jp form stress term of Mellor $(2003,2005)$ has a continuous vertical profile that, according to Ardhuin et al. (2008a), is valid as long as the bottom is flat. However the expression for the depth-dependent form stress term has been changed in Mellor (2008, 2011a,b) to include a Dirac delta function at the sea surface.

Bennis and Ardhuin (2011, hereafter BA11) criticized the delta function of Mellor (2008, 2011a,b) and suggested using wave-averaged momentum equations derived from the three-dimensional Lagrangian mean framework of Andrews and McIntyre (1978, hereafter AM78) with a wave-induced pressure term derived by Ardhuin et al. (2008b, hereafter ARB08) and given by Eq. (39) on page 45 of their paper. An advantage of the equation system of ARB08 and BA11 is that it is applicable to circulation over a sloping bottom. However, neither ARB08 nor BA11 have shown how to rederive an equivalent to their equation system including the wave-induced pressure term (as well as the vortex force term) by taking the average of the equations written in the coordinate system used by Mellor $(2003,2005)$. 
Therefore, in the community, there remains uncertainty concerning (i) the inapplicability of Mellor $(2003,2005)$ to a sloping bottom, (ii) the delta function of Mellor (2008, 2011a,b), and (iii) existing difficulty to see consistency between momentum equations that have been wave-averaged in the three-dimensional Lagrangian coordinates, on the one hand, and in the coordinates of Mellor on the other. Points (i)-(iii) are the subject of this study.

Generalizing the results of Mellor $(2003,2005)$ and Broström et al. (2008), Aiki and Greatbatch (2012, hereafter AG12) have developed depth-dependent equations for surface gravity waves and circulation in a vertically Lagrangian and horizontally Eulerian (VL) coordinate system. This framework, based on a thickness-weightedmean (TWM) approach, allows for a concise treatment of the thin viscous boundary layer at the sea surface, the incompressibility condition for circulation, and the energy interactions between waves and circulation. Recently Aiki and Greatbatch (2012, manuscript submitted to J. Phys. Oceanogr., hereafter AGVF) have developed an exact recipe to derive the Craik and Leibovich (1976, hereafter CL76) momentum equations by taking the wave average of equations written in the VL coordinates. The present manuscript is partly based on the recipe of AGVF but, rather than focus on the vortex force, we focus instead on the depth-dependent version of the radiation stress in LHS64.

The plan of the paper is as follows. The governing equations are explained in section 2. Then a discussion follows in section 3 concerning the different scalings of the wave-averaged equations that apply in different studies. We note that the depth-integrated radiation stress of LHS64 has been written as the product of firstorder waves in terms of a perturbation expansion and, thus, should be compared with the wave-induced pressure term in ARB08 (i.e., the wave setup/setdown term) rather than the vortex force. Our approach is complementary to that in Smith (2006) and Lane et al. (2007, section 4) who made comparisons between different versions of the depth-integrated momentum equations applicable to an inner shelf zone. Then, from section 4 onward, we focus on the scaling appropriate to LHS64 and develop the depth-dependent radiation stress term in the VL framework. We show that the wave-averaged momentum equations become applicable to a sloping bottom when written for the development of the Eulerian mean velocity rather than the Lagrangian mean velocity, a result that is a reexplanation of Ardhuin et al. (2008a) using the TWM theory. In section 5 we link our results to those of other studies, in particular LHS64, Smith (2006), ARB08, and the papers by Mellor. Finally, section 6 provides a summary and brief discussion. Overall, the present manuscript and AGVF, taken together, illustrate the consistency between momentum equations that have been wave averaged in the three-dimensional Lagrangian coordinates on the one hand and in the VL coordinates on the other.

\section{Governing equations}

We consider incompressible inviscid water of constant, uniform density in a nonrotating frame. We use the equations of AG12, which are briefly explained for convenience in appendix A of the present manuscript. It should be noted that the equations have been nondimensionalized (see appendix A). The nondimensionalization is not essential but serves to simplify the mathematics.

\section{a. Thickness-weighted-mean momentum equations}

The incompressible condition and the momentum equations in the VL coordinates, $(x, y, z, t)$, of AG12 are

$$
\begin{gathered}
\left(z_{z}^{c}\right)_{t}+\nabla \cdot\left(z_{z}^{c} \mathbf{V}\right)+\left(z_{z}^{c} w^{*}\right)_{z}=0 \\
\left(\partial_{t}+\mathbf{V} \cdot \boldsymbol{\nabla}+w^{*} \partial_{z}\right) z^{c}=w, \\
\left(\partial_{t}+\mathbf{V} \cdot \nabla+w^{*} \partial_{z}\right) \mathbf{V}=-\nabla(p+\eta)+p_{z^{c}} \nabla z^{c}, \\
\left(\partial_{t}+\mathbf{V} \cdot \nabla+w^{*} \partial_{z}\right) w=-p_{z^{c}},
\end{gathered}
$$

where $z^{c}=z^{c}(x, y, z, t)$ is the instantaneous height of fluid particles in the standard Eulerian-Cartesian coordinates. The vertical coordinate $z$ is a low-pass filtered height coordinate (see appendix A) and $z_{z}^{c}$ is the thickness. ${ }^{1}$ The horizontal coordinates $x$ and $y$ are the same as the Eulerian-Cartesian coordinates. The quantity $\mathbf{V}$ is the horizontal velocity vector, $w$ is the vertical component of velocity, $w^{*}$ represents water flux through the surfaces of fixed $z, \nabla \equiv\left(\partial_{x}, \partial_{y}\right)$ is the lateral gradient operator along the surfaces of fixed $z$, and $\nabla z=0$ is understood. The quantity $p$ is the sum of the oceanic nonhydrostatic and atmospheric pressure, and $\eta$ is the instantaneous sea surface height. Throughout this paper, we shall assume $p=0$ at the surface, corresponding to assuming uniform atmospheric pressure at the surface. Table 1 presents a list of the symbols used in the text.

The momentum equations (1c)-(1d) can be written in a flux-divergence form as

\footnotetext{
${ }^{1}$ The vertical coordinate $z$ in the present study corresponds roughly to $\zeta$ in Mellor (2003), s in his later studies, and $Z$ in Broström et al. (2008).
} 
TABLE 1 . List of symbols, where $A$ is an arbitrary quantity.

\begin{tabular}{|c|c|}
\hline$\left(x^{c}, y^{c}, z^{c}, t^{c}\right)$ & Eulerian-Cartesian coordinates \\
\hline$(x, y, z, t)$ & Vertically Lagrangian and horizontally Eulerian (VL) coordinates \\
\hline $\bar{A}^{c}$ & Time-mean in Eulerian-Cartesian coordinates \\
\hline$\hat{A} \equiv \overline{z_{z}^{c} A}$ & Thickness-weighted time-mean in the VL coordinates \\
\hline $\bar{A}$ & Unweighted time-mean in the VL coordinates \\
\hline$A^{\prime} \equiv A-\bar{A}^{c}$ & Deviation from the Eulerian mean, compared at fixed $z^{c}\left({\overline{A^{\prime}}}^{c}=0\right)$ \\
\hline$A^{\prime \prime} \equiv A-\hat{A}$ & Deviation from the thickness-weighted mean, compared at fixed $z\left(\overline{z_{z}^{c} A^{\prime \prime}}=0\right)$ \\
\hline$A^{\prime \prime \prime} \equiv A-\bar{A}$ & Deviation from the unweighted mean, compared at fixed $z\left(\overline{A^{\prime \prime \prime}}=0\right)$ \\
\hline$\nabla^{c} \equiv\left(\partial_{x^{c}}, \partial_{y^{c}}\right)$ & Horizontal gradient in Eulerian-Cartesian coordinates $\left[\nabla^{c}=\nabla-\left(\nabla z^{c}\right) \partial_{z^{c}}\right]$ \\
\hline$\nabla \equiv\left(\partial_{x}, \partial_{y}\right)$ & Lateral gradient in the VL coordinates $\left(\nabla z=0, \nabla z^{c}=\nabla z^{\prime \prime \prime}\right)$ \\
\hline $\mathbf{V} \equiv(u, v)$ & Horizontal component of velocity \\
\hline$w$ & Vertical component of velocity \\
\hline$w^{*} \equiv\left(w-z_{t}^{c}-\mathbf{V} \cdot \nabla z^{c}\right) / z_{z}^{c}$ & Vertical velocity associated with volume flux through surface of fixed $z$ \\
\hline$(\hat{\mathbf{V}}, \hat{w})$ & TWM velocity \\
\hline$\left(\hat{\mathbf{V}}, \widehat{w^{*}}\right)$ & Total transport velocity $\left(\boldsymbol{\nabla} \cdot \hat{\mathbf{V}}+\widehat{w_{z}^{*}}=0\right)$ \\
\hline$\eta$ & Sea surface height \\
\hline$p$ & Sum of oceanic nonhydrostatic pressure and atmospheric sea surface pressure \\
\hline$p^{\mathrm{Mel}} \equiv p+\eta-z^{c}$ & Combined nonhydrostatic and hydrostatic pressure in papers by Mellor \\
\hline $\mathcal{F} \mathcal{S}^{\mathbf{V}}$ & Divergence of form stress $\equiv-\left[\overline{z^{\prime \prime \prime} \nabla\left(p^{\prime \prime \prime}+\eta^{\prime \prime \prime}\right)}\right]_{z}+\nabla\left(\frac{1}{z^{\prime \prime \prime} p_{z}^{\prime \prime \prime}}\right)$ \\
\hline $\mathcal{R S}^{A}$ for $A=u, v$, and $w$ & Divergence of the Reynolds stress $\equiv \nabla \cdot\left(\overline{z_{z}^{c} \mathbf{V}^{\prime \prime} A^{\prime \prime}}\right)+\left(\overline{z_{z}^{c} w^{* \prime \prime} A^{\prime \prime}}\right)_{z}$ \\
\hline$h$ & Bottom depth $(>0)$ \\
\hline $\mathcal{A}$ & Amplitude of $O(\alpha)$ wave \\
\hline$\alpha$ & Surface slope of $O(\alpha)$ wave \\
\hline$\kappa \equiv \sqrt{k^{2}+l^{2}}$ & Horizontal wavenumber of $O(\alpha)$ wave \\
\hline$\sigma$ & Frequency of $O(\alpha)$ wave \\
\hline$\theta \equiv k x+l y-\sigma \tau$ & Phase of $O(\alpha)$ wave \\
\hline$\partial_{\tau}$ & Time derivative operator for wave quantities \\
\hline$\partial_{T}$ & Time derivative operator for mean quantities $\left(\partial_{t}=\partial_{\tau}+\alpha \partial_{T}\right)$ \\
\hline$\dot{\nabla}$ & Lateral gradient for wave quantities \\
\hline $\bar{\nabla}$ & Lateral gradient for mean quantities $(\boldsymbol{\nabla}=\dot{\boldsymbol{\nabla}}+\alpha \overline{\boldsymbol{\nabla}})$ \\
\hline $\mathbf{V}_{2}^{q s} \equiv\left(\overline{z_{1}^{\prime \prime \prime} \mathbf{V}_{1}^{\prime \prime \prime}}\right)_{z}$ & Horizontal component of the quasi-Stokes velocity at $O\left(\alpha^{2}\right)$ \\
\hline$w_{3}^{q s}=-\bar{\nabla} \cdot\left(\overline{z_{1}^{\prime \prime \prime} \mathbf{V}_{1}^{\prime \prime \prime}}\right)$ & Vertical component of the quasi-Stokes velocity at $O\left(\alpha^{3}\right)$ \\
\hline$\overline{z_{1 z}^{\prime \prime \prime} \mathbf{V}_{1}^{\prime \prime \prime}}-\overline{w_{1}^{\prime \prime \prime}} \dot{\mathbf{\nabla}} z_{1}^{\prime \prime \prime}$ & Horizontal component of the pseudomomentum in the VL framework (cf. AGVF) \\
\hline$E_{2} \equiv \frac{1}{2} \int_{-h}^{\bar{\eta}} \overline{\left|\mathbf{V}_{1}^{\prime \prime \prime}\right|^{2}+w_{1}^{\prime \prime \prime 2}} d z+\frac{1}{2} \overline{\eta_{1}^{\prime \prime \prime}}$ & Depth-integrated total (kinetic plus potential) wave energy at $O\left(\alpha^{2}\right)$ \\
\hline
\end{tabular}

$\left(z_{z}^{c} \mathbf{V}\right)_{t}+\nabla \cdot\left(z_{z}^{c} \mathbf{V V}\right)+\left(z_{z}^{c} w^{*} \mathbf{V}\right)_{z}=-z_{z}^{c} \nabla(p+\eta)+p_{z} \nabla z^{c}$

$\left(z_{z}^{c} w\right)_{t}+\nabla \cdot\left(z_{z}^{c} \mathbf{V} w\right)+\left(z_{z}^{c} w^{*} w\right)_{z}=-p_{z}$

Low-pass temporal filtering each of (1a), (2a), and (2b) yields thickness-weighted-mean (TWM) equations for incompressibility and the horizontal and vertical components of momentum,

$$
\begin{aligned}
& \nabla \cdot \hat{\mathbf{V}}+\widehat{w_{z}^{*}}=0 \\
&\left.\hat{\mathbf{V}}_{t}+\nabla \cdot(\hat{\mathbf{V}} \hat{\mathbf{V}})+\widehat{\left(w^{*} \hat{\mathbf{V}}\right.}\right)_{z}+\mathcal{R} \mathcal{S}^{\mathbf{V}}=-\nabla(\bar{p}+\bar{\eta})+\mathcal{F S}^{\mathbf{V}} \\
& \hat{w}_{t}+\nabla \cdot(\hat{\mathbf{V}} \hat{w})+\left(\widehat{w^{*} \hat{w}}\right)_{z}+\mathcal{R S}^{w}=-\bar{p}_{z}
\end{aligned}
$$

where $\overline{z_{z}^{c}} \equiv 1$ (since $\overline{z^{c}} \equiv z$, following from the definition of $z$ ) has been used and the caret symbol is the TWM operator $\left(\hat{A} \equiv \overline{z_{z}^{c} A}\right.$ for an arbitrary quantity $\left.A\right)$. The velocity variable $\left(\hat{\mathbf{V}}, \widehat{w^{*}}\right)$, which satisfies the incompressibility condition (3a), is called the total transport velocity. The total transport velocity corresponds to the Lagrangian mean velocity in the three-dimensional Lagrangian mean framework. The symbols $\mathcal{R S} \mathcal{S}^{\mathbf{V}}$ and $\mathcal{R} \mathcal{S}^{w}$ in (3b)-(3c) are the Reynolds stress terms defined by

$$
\mathcal{R S}^{A} \equiv \nabla \cdot\left(\overline{z_{z}^{c} \mathbf{V}^{\prime \prime} A^{\prime \prime}}\right)+\left(\overline{z_{z}^{c} w^{* \prime \prime} A^{\prime \prime}}\right)_{z}
$$

for $A=u, v$, and $w$, and the double-prime symbol is the deviation from the TWM $\left(A^{\prime \prime} \equiv A-\hat{A}\right.$, compared at fixed $z$ ). The vertical flux of momentum in (4a) is given in terms of $w^{* \prime \prime}$ (the vertical velocity in the low-pass filtered coordinate, $z$, not $\left.w^{\prime \prime}\right)$ with the consequence that it is small (see AG12 for details). The symbol $\mathcal{F S}^{\mathbf{V}}$ in (3b) is the form stress term defined by

$$
\begin{aligned}
\mathcal{F} \mathcal{S}^{\mathbf{V}} & \equiv-\overline{z_{z}^{\prime \prime \prime} \nabla\left(p^{\prime \prime \prime}+\eta^{\prime \prime \prime}\right)}+\overline{p_{z}^{\prime \prime \prime} \nabla z^{\prime \prime \prime}} \\
& =-\left[\overline{z^{\prime \prime \prime} \nabla\left(p^{\prime \prime \prime}+\eta^{\prime \prime \prime}\right)}\right]_{z}+\nabla\left(\overline{z^{\prime \prime \prime} p_{z}^{\prime \prime \prime}}\right),
\end{aligned}
$$


where the triple-prime symbol is the deviation from the unweighted mean $\left(A^{\prime \prime \prime} \equiv A-\bar{A}\right.$, compared at fixed $\left.z\right)$, in particular $z^{\prime \prime \prime} \equiv z^{c}-z$ is noted. ${ }^{2}$

Moving $\mathcal{F} \mathcal{S}^{\mathbf{V}}$ to the lhs of (3b) yields the depthdependent radiation stress term, $\mathcal{R} \mathcal{S}^{\mathbf{v}}-\mathcal{F} \mathcal{S}^{\mathbf{V}}$.

\section{b. Kinematic boundary condition}

As illustrated in Fig. 1, the sea surface is referred to as $z^{c}=\eta$ in the Eulerian-Cartesian coordinates, whereas it is referred to as $z=\bar{\eta}$ in the VL coordinates. AG12 have shown that the kinematic boundary condition at the sea surface reads

$$
\begin{aligned}
& \widehat{w^{*}}=\bar{\eta}_{t}+\hat{\mathbf{V}} \cdot \nabla \bar{\eta}, \\
& w^{* \prime \prime}=\mathbf{V}^{\prime \prime} \cdot \nabla \bar{\eta}, \\
& \eta^{\prime \prime \prime} \equiv z^{\prime \prime \prime}
\end{aligned}
$$

at $z=\bar{\eta}$. Application of the explanation of AG12 to a sloping bottom yields (i.e., replace $z=\bar{\eta}$ in section $2 \mathrm{e}$ of AG12 with $z=-h$ ),

$$
\begin{aligned}
& \widehat{w^{*}}=-\hat{\mathbf{V}} \cdot \nabla h, \\
& w^{* \prime \prime}=-\mathbf{V}^{\prime \prime} \cdot \nabla h, \\
& z^{\prime \prime \prime}=0,
\end{aligned}
$$
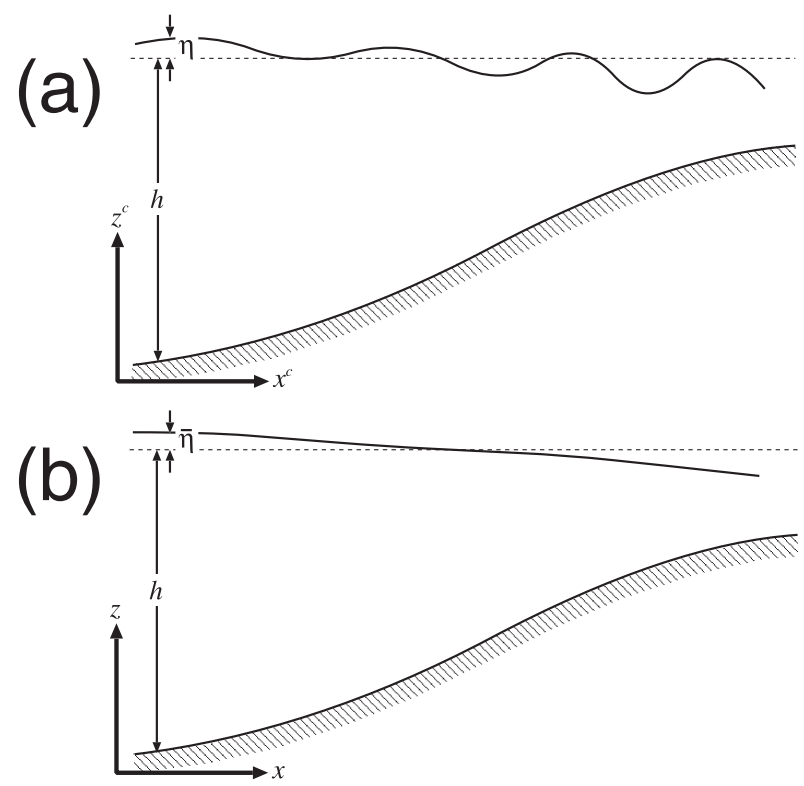

FIG. 1. Schematic of waves on a sloping bottom in (a) the Eulerian-Cartesian coordinate and (b) the vertically Lagrangian coordinate systems.

at $z=-h$. That $z^{\prime \prime \prime}=0$ at the bottom has already been shown in the mesoscale eddy literature (e.g., McDougall and McIntosh 2001).

The depth integral of the Reynolds stress term (4a) is

$$
\begin{aligned}
\int_{-h}^{\bar{\eta}} \mathcal{R} \mathcal{S}^{A} d z & =\int_{-h}^{\bar{\eta}} \nabla \cdot\left(\overline{z_{z}^{c} \mathbf{V}^{\prime \prime} A^{\prime \prime}}\right) d z+\left.\left(\overline{z_{z}^{c} w^{* \prime \prime} A^{\prime \prime}}\right)\right|_{z=\bar{\eta}}-\left.\left(\overline{z_{z}^{c} w^{* \prime \prime} A^{\prime \prime}}\right)\right|_{z=-h} \\
& =\int_{-h}^{\bar{\eta}} \nabla \cdot\left(\overline{z_{z}^{c} \mathbf{V}^{\prime \prime} A^{\prime \prime}}\right) d z+\left.\left(\overline{z_{z}^{c} \mathbf{V}^{\prime \prime} A^{\prime \prime}} \cdot \nabla \bar{\eta}\right)\right|_{z=\bar{\eta}}+\left.\left(\overline{z_{z}^{c} \mathbf{V}^{\prime \prime} A^{\prime \prime}} \cdot \nabla h\right)\right|_{z=-h}=\nabla \cdot \int_{-h}^{\bar{\eta}}\left(\overline{z_{z}^{c} \mathbf{V}^{\prime \prime} A^{\prime \prime}}\right) d z,
\end{aligned}
$$

where (5b) and (6b) have been used to derive the second line. The depth integral of the form stress term (4b) is

$$
\begin{aligned}
& \int_{-h}^{\bar{\eta}} \mathcal{F} \mathcal{S}^{\mathbf{V}} d z=-\overline{\eta^{\prime \prime \prime} \nabla\left(\left.p^{\prime \prime \prime}\right|_{z=\bar{\eta}}+\eta^{\prime \prime \prime}\right)}+\int_{-h}^{\bar{\eta}} \nabla\left(\overline{z^{\prime \prime \prime} p_{z}^{\prime \prime \prime}}\right) d z=-\overline{\eta^{\prime \prime \prime} \nabla\left(\left.p^{\prime \prime \prime}\right|_{z=\bar{\eta}}+\eta^{\prime \prime \prime}\right)}+\boldsymbol{\nabla} \int_{-h}^{\bar{\eta}}\left(\overline{z^{\prime \prime \prime} p_{z}^{\prime \prime \prime}}\right) d z-\left(\overline{\left.\eta^{\prime \prime \prime} p_{z}^{\prime \prime \prime}\right|_{z=\bar{\eta}}}\right) \nabla \bar{\eta} \\
& =-\frac{1}{2} \nabla \overline{\eta^{\prime \prime \prime}}+\nabla \int_{-h}^{\bar{\eta}}\left(\overline{z^{\prime \prime \prime} p_{z}^{\prime \prime \prime}}\right) d z-\left.\overline{\eta^{\prime \prime \prime}\left(\nabla p^{\prime \prime \prime}+p_{z}^{\prime \prime \prime} \nabla \bar{\eta}\right)}\right|_{z=\bar{\eta}}
\end{aligned}
$$

where $(6 \mathrm{c})$ has been used to derive the first line.

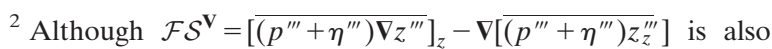
true, the expression as in the last line of $(4 b)$ is convenient for handling the bottom boundary condition where (6c) holds, resulting in $\overline{z^{\prime \prime \prime} \nabla\left(p^{\prime \prime \prime}+\eta^{\prime \prime \prime}\right)}=0$ at $z=-h$ no matter how the bottom is sloped.
}

\section{Scaling the low-pass filtered equations}

Let $\alpha \ll 1$ be the scale for the surface slope. In this manuscript, we assume that the bottom slope is $\nabla h \sim \alpha$, with a consequence, as we note below, that the aspect ratio of the circulation is also scaled by $\alpha$. Then, let the horizontal gradient operator be written as $\boldsymbol{\nabla}=\dot{\boldsymbol{\nabla}}+\alpha \overline{\boldsymbol{\nabla}}$ where $\left(\partial_{z} \sim \dot{\nabla}\right.$ has been used and) $\dot{\nabla}$ operates on wave 
quantities and $\bar{\nabla}$ operates on the low-pass filtered quantities as well as on the large spatial-scale variation of the wave quantities. Note that $\nabla \bar{A}=\alpha \bar{\nabla} \bar{A}$ and $\nabla A^{\prime \prime \prime}=$ $\dot{\nabla} A^{\prime \prime \prime}+\alpha \bar{\nabla} A^{\prime \prime \prime}$ for an arbitrary quantity $A$.

\section{a. Perturbation expansion}

The fluctuation component of all quantities is expanded from $O(\alpha)$, except for $w^{* \prime \prime \prime}$, which is expanded from $O\left(\alpha^{2}\right)$,

$$
\begin{aligned}
& z^{\prime \prime \prime}=\alpha z_{1}^{\prime \prime \prime}+\alpha^{2} z_{2}^{\prime \prime \prime}+O\left(\alpha^{3}\right), \\
& \eta^{\prime \prime \prime}=\alpha \eta_{1}^{\prime \prime \prime}+\alpha^{2} \eta_{2}^{\prime \prime \prime}+O\left(\alpha^{3}\right), \\
& p^{\prime \prime \prime}=\alpha p_{1}^{\prime \prime \prime}+\alpha^{2} p_{2}^{\prime \prime \prime}+O\left(\alpha^{3}\right), \\
& \mathbf{V}^{\prime \prime \prime}=\alpha \mathbf{V}_{1}^{\prime \prime \prime}+\alpha^{2} \mathbf{V}_{2}^{\prime \prime \prime}+O\left(\alpha^{3}\right), \\
& w^{\prime \prime \prime}=\alpha w_{1}^{\prime \prime \prime}+\alpha^{2} w_{2}^{\prime \prime \prime}+O\left(\alpha^{3}\right), \\
& w^{* \prime \prime \prime}=\alpha^{2} w_{2}^{* \prime \prime \prime}+O\left(\alpha^{3}\right) .
\end{aligned}
$$

The scaling of $w^{* \prime \prime \prime}$ stems from the scaling of the bottom slope. Writing (6b) at second order in $\alpha$ yields $w_{2}^{* \prime \prime}=$ $-\mathbf{V}_{1}^{\prime \prime} \cdot \bar{\nabla} h\left(\text { or } w_{2}^{* \prime \prime \prime}=-\mathbf{V}_{1}^{\prime \prime \prime} \cdot \bar{\nabla} h \text { because } \mathbf{V}_{1}^{\prime \prime \prime}=\mathbf{V}_{1}^{\prime \prime}\right)^{3}$ Therefore the perturbation expansion for $w^{* \prime \prime \prime}$ starts from $O\left(\alpha^{2}\right)$.

\section{b. Longuet-Higgins and Stewart versus the vortex force}

The depth-integrated radiation stress of LHS64 is written in terms of the product of $O(\alpha)$ wave quantities. It follows that the depth-integrated Reynolds and form stress terms (7a)-(7b) appear at $O\left(\alpha^{3}\right)$ and can be written as,

$$
\begin{aligned}
& \int_{-h}^{\bar{\eta}} \mathcal{R S}_{3}^{\mathbf{V}} d z=\bar{\nabla} \cdot \int_{-h}^{\bar{\eta}} \overline{\mathbf{V}_{1}^{\prime \prime \prime} \mathbf{V}_{1}^{\prime \prime \prime}} d z, \\
& \int_{-h}^{\bar{\eta}} \mathcal{F} \mathcal{S}_{3}^{\mathbf{V}} d z=-\frac{1}{2} \bar{\nabla} \overline{\eta_{1}^{\prime \prime \prime}}+\bar{\nabla} \int_{-h}^{\bar{\eta}}\left(\overline{z_{1}^{\prime \prime \prime}} p_{1 z}^{\prime \prime \prime}\right) d z,
\end{aligned}
$$

where we have used $p^{\prime \prime \prime}=0$ at $z=\bar{\eta}$. Note that it is the use of the $\bar{\nabla}$ operator that makes these expressions third order in $\alpha$. Equations (9a)-(9b) can also be derived by taking the depth-integral of $(4 a)-(4 b)$ written at $O\left(\alpha^{3}\right)$,

\footnotetext{
${ }^{3} A_{1}^{\prime \prime}=\left(A-\overline{z_{z}^{c} A}\right)_{1}=\left(A-\bar{A}-\overline{z_{z}^{\prime \prime \prime} A^{\prime \prime \prime}}\right)_{1}=\left(A^{\prime \prime \prime}-\overline{z_{z}^{\prime \prime \prime} A^{\prime \prime \prime}}\right)_{1}=A_{1}^{\prime \prime \prime}$ for an arbitrary quantity $A$. The numeric subscript attached to the brackets in the present study represents summation of terms at a given order of $\alpha$.
}

$$
\begin{aligned}
\mathcal{R S}_{3}^{\mathbf{V}=} & \bar{\nabla} \cdot\left(\overline{\mathbf{V}_{1}^{\prime \prime \prime} \mathbf{V}_{1}^{\prime \prime \prime}}\right)+\left(\overline{w_{2}^{* \prime \prime \prime}} \mathbf{V}_{1}^{\prime \prime \prime}\right)_{z} \\
\mathcal{F S}_{3}^{\mathbf{V}=} & -\left[\overline{z_{2}^{\prime \prime} \dot{\mathbf{V}}\left(p_{1}^{\prime \prime \prime}+\eta_{1}^{\prime \prime \prime}\right)}\right]_{z}-\left[\overline{z_{1}^{\prime \prime \prime} \dot{\mathbf{\nabla}}\left(p_{2}^{\prime \prime \prime}+\eta_{2}^{\prime \prime \prime}\right)}\right]_{z} \\
& -\left[\overline{z_{1}^{\prime \prime \prime} \bar{\nabla}\left(p_{1}^{\prime \prime \prime}+\eta_{1}^{\prime \prime \prime}\right)}\right]_{z}+\bar{\nabla}\left(\overline{z_{1}^{\prime \prime \prime} p_{1 z}^{\prime \prime \prime}}\right)
\end{aligned}
$$

where the third term, $\overline{z_{1}^{\prime \prime \prime} \bar{\nabla}\left(p_{1}^{\prime \prime \prime}+\eta_{1}^{\prime \prime \prime}\right)}$, on the rhs of (10b) should not be confused with $\overline{z_{1}^{\prime \prime \prime} \dot{\nabla}\left(p_{1}^{\prime \prime \prime}+\eta_{1}^{\prime \prime \prime}\right)}$ [the latter is $O\left(\alpha^{2}\right)$ ]. It should be noted that, in contrast to the depth-integrated terms (9a)-(9b), the depthdependent terms (10a)-(10b) contain quantities associated with $O\left(\alpha^{2}\right)$ waves.

Both Reynolds and form stress terms (10a)-(10b) are part of the horizontal component of the TWM momentum equations (3b) to be written at $O\left(\alpha^{3}\right)$. We now come to the question of how to scale the mean flow. The first choice is suitable for the circulation within an inner shelf zone where the depth-integrated cancellation between the Eulerian mean velocity and the Stokes-drift velocity might occur (cf. Lentz and Fewings 2012). We therefore put

$$
\begin{gathered}
\overline{\mathbf{v}}=\alpha^{2} \overline{\mathbf{v}}_{2}+O\left(\alpha^{3}\right), \\
\overline{w^{*}}=\alpha^{3} \overline{w_{3}^{*}}+O\left(\alpha^{4}\right), \\
\bar{\eta}=\alpha^{2} \bar{\eta}_{2}+O\left(\alpha^{3}\right), \\
\bar{p}=\alpha^{2} \bar{p}_{2}+O\left(\alpha^{3}\right),
\end{gathered}
$$

so that the magnitude of the horizontal component of the mean velocity is $O\left(\alpha^{2}\right)$ and is the same order as the Stokes-drift velocity. The TWM equation system (3a)-(3c) becomes,

$$
\begin{aligned}
\bar{\nabla} \cdot \hat{\mathbf{V}}_{2}+\widehat{w_{3 z}^{*}} & =0, \\
\partial_{T} \hat{\mathbf{V}}_{2}+\mathcal{R} \mathcal{S}_{3}^{\mathbf{V}} & =-\bar{\nabla}\left(\bar{p}_{2}+\bar{\eta}_{2}\right)+\mathcal{F} \mathcal{S}_{3}^{\mathbf{V}}, \\
0 & =-\bar{p}_{2 z},
\end{aligned}
$$

where the time development of low-pass filtered quantities is assumed to be one order slower than the phase cycle of the waves (i.e., $\partial_{t}=\partial_{\tau}+\alpha \partial_{T}$ where $\partial_{\tau}$ operates on wave quantities and $\partial_{T}$ operates on the low-pass filtered quantities as well as the slow time evolution of the wave quantities). The horizontal momentum Eq. (12b) has been written at $O\left(\alpha^{3}\right)$, and excludes a mean-flow advection term, such as $\hat{\mathbf{V}}_{2} \cdot \bar{\nabla} \hat{\mathbf{V}}_{2}$, because it is $O\left(\alpha^{5}\right)$.

The second choice for the scaling is suitable for circulation driven by a strong wind, 
TABLE 2. Comparison of the scalings of the low-pass-filtered equations given in section 3 of the present manuscript, MRL04, and CL76. Underlined quantities indicate that the vortex force is of higher order in $\alpha$ than the corresponding wave-averaged momentum equations.

\begin{tabular}{lccccc}
\hline \hline & \multicolumn{3}{c}{$\begin{array}{c}\text { The present } \\
\text { manuscript }\end{array}$} \\
\cline { 2 - 4 } \multicolumn{1}{c}{ Equation system } & $(12 \mathrm{a}-\mathrm{c})$ & $(14 \mathrm{a}-\mathrm{c})$ & $(15 \mathrm{a}-\mathrm{c})$ & & \\
\hline Coefficient of $\partial_{T}$ & $\alpha$ & $\alpha^{2}$ & $\alpha^{3}$ & $\alpha^{4}$ & $\alpha^{2}$ \\
Coefficient of $\overline{\boldsymbol{\nabla}}$ & $\alpha$ & $\alpha$ & $\alpha$ & $\alpha^{2}$ & 1 \\
$\hat{\mathbf{V}}, \overline{\mathbf{V}}, \overline{\mathbf{V}}^{c}$ & $\alpha^{2}$ & $\alpha$ & $\alpha^{2}$ & $\alpha^{2}$ & $\alpha^{2}$ \\
$\widehat{w^{*}}, \bar{w}^{*}, \bar{w}, \bar{w}^{c}$ & $\alpha^{3}$ & $\alpha^{2}$ & $\alpha^{3}$ & $\alpha^{4}$ & $\alpha^{2}$ \\
Horizontal momentum & $\alpha^{3}$ & $\alpha^{3}$ & $\alpha^{5}$ & $\alpha^{6}$ & $\alpha^{4}$ \\
$\quad$ equation & & & & & \\
Vertical momentum & $\alpha^{2}$ & $\alpha^{2}$ & $\alpha^{4}$ & $\alpha^{4}$ & $\alpha^{4}$ \\
$\quad$ equation & & & & & \\
$\mathbf{V}^{\text {Stokes }} \times\left(\boldsymbol{\nabla} \times \overline{\mathbf{V}}^{c}\right)$ & $\underline{\alpha}^{5}$ & $\underline{\alpha}^{4}$ & $\alpha^{5}$ & $\alpha^{6}$ & $\alpha^{4}$ \\
$\mathbf{V}^{\text {Stokes }} \cdot\left(\partial_{z} \overline{\mathbf{V}}^{c}-\bar{\nabla}^{c}\right)$ & $\underline{\alpha}^{4}$ & $\underline{\alpha}^{3}$ & $\alpha^{4}$ & $\alpha^{4}$ & $\alpha^{4}$ \\
\hline
\end{tabular}

$$
\begin{gathered}
\overline{\mathbf{v}}=\alpha \overline{\mathbf{V}}_{1}+O\left(\alpha^{2}\right), \\
\overline{w^{*}}=\alpha^{2} \overline{w_{2}^{*}}+O\left(\alpha^{3}\right), \\
\bar{\eta}=\alpha^{2} \bar{\eta}_{2}+O\left(\alpha^{3}\right), \\
\bar{p}=\alpha^{2} \bar{p}_{2}+O\left(\alpha^{3}\right) .
\end{gathered}
$$

The horizontal component of the mean velocity is now $O(\alpha)$, which is one order greater than the Stokes-drift velocity, and the TWM equation system (3a)-(3c) becomes

$$
\begin{aligned}
\overline{\mathbf{\nabla}} \cdot \hat{\mathbf{V}}_{1}+\widehat{w_{2 z}^{*}} & =0, \\
\left(\partial_{T}+\hat{\mathbf{V}}_{1} \cdot \bar{\nabla}+\widehat{w_{2}^{*}} \partial_{z}\right) \hat{\mathbf{V}}_{1}+\mathcal{R} \mathcal{S}_{3}^{\mathbf{V}} & =-\bar{\nabla}\left(\bar{p}_{2}+\bar{\eta}_{2}\right)+\mathcal{F} \mathcal{S}_{3}^{\mathbf{V}}, \\
0 & =-\bar{p}_{2 z},
\end{aligned}
$$

where $\hat{A}_{1}=\left(\bar{A}+\overline{z_{z}^{\prime \prime \prime} A^{\prime \prime \prime}}\right)_{1}=\bar{A}_{1}$ for an arbitrary quantity $A$, and the time development of the low-pass filtered quantities is assumed to be two orders slower than the phase cycle of the waves (i.e., $\partial_{t}=\partial_{\tau}+\alpha^{2} \partial_{T}$ ). In both systems associated with the first and second choices for the scaling, the horizontal momentum equations (12b) and (14b) are written at $O\left(\alpha^{3}\right)$ whereas the horizontal component of the vortex force is $O\left(\alpha^{5}\right)$ or $O\left(\alpha^{4}\right)$, as noted by the underlined quantities in Table $2 .{ }^{4}$ Likewise

\footnotetext{
${ }^{4}$ The horizontal and vertical components of the vortex force are written by $\mathbf{V}^{\text {Stokes }} \times\left(\boldsymbol{\nabla} \times \overline{\mathbf{V}}^{c}\right)$ and $\mathbf{V}^{\text {Stokes }} \cdot\left(\partial_{z} \overline{\mathbf{V}}^{c}-\nabla \bar{w}^{c}\right)$, respectively, where $\mathbf{V}^{\text {Stokes }}$ is the Stokes-drift velocity (CL76; Craik 1985).
}

the vertical momentum equations (12c) and (14c) are both $O\left(\alpha^{2}\right)$ whereas the vertical component of the vortex force is $O\left(\alpha^{4}\right)$ or $O\left(\alpha^{3}\right)$. Consequently, for the choice of scalings presented so far, the vortex force equations are not applicable, and this is true even if the momentum equations are rewritten for the development of the Eulerian mean velocity.

To compare with the circulation regime associated with the vortex force, we consider a third choice for the scaling, which is the same as that in (12a)-(12c) except that the time development of low-pass filtered quantities is assumed to be three orders slower than the phase cycle of the waves (i.e., $\partial_{t}=\partial_{\tau}+\alpha^{3} \partial_{T}$ ). The TWM equation system (3a)-(3c) becomes

$$
\begin{aligned}
& \bar{\nabla} \cdot \hat{\mathbf{V}}_{2}+\widehat{w_{3 z}^{*}}=0, \\
&\left(\partial_{T}+\hat{\mathbf{V}}_{2} \cdot \bar{\nabla}+\widehat{w_{3}^{*}} \partial_{z}\right) \hat{\mathbf{V}}_{2}+\mathcal{R S}_{5}^{\mathbf{V}}=-\bar{\nabla}\left(\bar{p}_{4}+\bar{\eta}_{4}\right)+\mathcal{F S}_{5}^{\mathbf{V}}, \\
& \mathcal{R} \mathcal{S}_{4}^{w}=-\bar{p}_{4 z},
\end{aligned}
$$

where the horizontal and vertical momentum equations now appear at $O\left(\alpha^{5}\right)$ and $O\left(\alpha^{4}\right)$, respectively. The Reynolds stress and the form stress terms are given by,

$$
\begin{aligned}
& \mathcal{R S}_{5}^{\mathbf{V}}=\bar{\nabla} \cdot\left(\overline{\mathbf{V}^{\prime \prime} \mathbf{V}^{\prime \prime}}\right)_{4}+\bar{\nabla} \cdot\left(\overline{z_{z}^{\prime \prime \prime} \mathbf{V}^{\prime \prime} \mathbf{V}^{\prime \prime}}\right)_{4} \\
& +\left(\overline{w^{* \prime \prime} \mathbf{V}^{\prime \prime}}\right)_{5 z}+\left(\overline{z_{z}^{\prime \prime \prime} w^{* \prime \prime} \mathbf{V}^{\prime \prime}}\right)_{5 z} \text {, } \\
& \mathcal{F}_{5} \mathbf{V}=-\left[\overline{z^{\prime \prime \prime} \dot{\nabla}\left(p^{\prime \prime \prime}+\eta^{\prime \prime \prime}\right)}\right]_{5 z}-\left[\overline{z^{\prime \prime \prime} \bar{\nabla}\left(p^{\prime \prime \prime}+\eta^{\prime \prime \prime}\right)}\right]_{5 z} \\
& +\bar{\nabla}\left(\overline{z^{\prime \prime \prime} p_{z}^{\prime \prime \prime}}\right)_{4} \text {, } \\
& \mathcal{R} \mathcal{S}_{4}^{w}=\bar{\nabla} \cdot\left(\overline{\mathbf{V}^{\prime \prime} w^{\prime \prime}}\right)_{3}+\bar{\nabla} \cdot\left(\overline{z_{z}^{\prime \prime \prime} \mathbf{V}^{\prime \prime} w^{\prime \prime}}\right)_{3} \\
& +\left(\overline{w^{* \prime \prime} w^{\prime \prime}}\right)_{4 z}+\left(\overline{z_{z}^{\prime \prime \prime} w^{* \prime \prime} w^{\prime \prime}}\right)_{4 z} \text {, }
\end{aligned}
$$

where the numeric subscript attached to the brackets represents summation of terms at a given order of $\alpha$ (see Table 3 for a template). Because the depth-dependent stress terms (15d)-(15f) consist of waves up to $O\left(\alpha^{4}\right)$, the depth-integral of (15d)-(15f) cannot be written as the product of $O(\alpha)$ wave quantities, in contrast to LHS64. Indeed, the Reynolds stress and form stress terms at lower order in $\alpha$ are now zero, and the scaling implied by LHS64 is no longer valid. Rather, the Reynolds stress and form stress terms $\mathcal{R} \mathcal{S}_{5}^{\mathbf{V}}$ and $\mathcal{F} \mathcal{S}_{5}^{\mathbf{V}}$ are of the same order as the horizontal component of the vortex force, and the stress term $\mathcal{R S}_{4}^{w}$ is of the same order as the vertical component of the vortex force, and both sets of terms can be transformed into the vortex force using the recipe of AGVF. Indeed, it is an important point that the Reynolds stress and form stress terms appear at 
TABLE 3. The rule of numeric subscript in the present study, which represents summation for a given order of perturbation expansion in terms of $\alpha$.

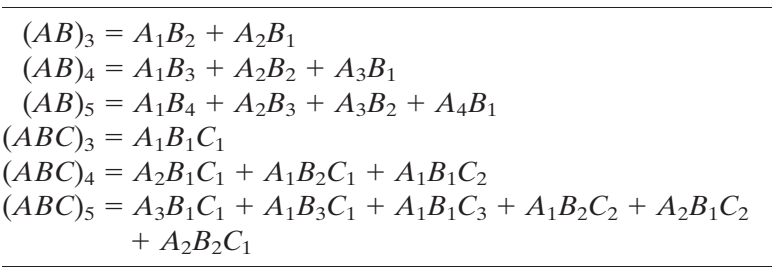

higher order in $\alpha$ in the scaling associated with the vortex force than in the scaling considered by LHS64. In fact, as shown in Table 2, the scaling of (15a)-(15c) is similar to that of McWilliams et al. (2004, hereafter MRL04).

To summarize, the scaling of the low-pass filtered equations associated with the radiation stress of LHS64 is described by either the equation system (12a)-(12c) or (14a)-(14c) and should not be confused with the scaling appropriate to the vortex force (see Lane et al. 2007). The fact that we focus in this paper on the scaling associated with the radiation stress of LHS64 is for the purpose of mathematical clarity regarding the attempt of Mellor $(2003,2008)$ to derive a depth-dependent version of LHS64 and does not necessarily indicate that the radiation stress (or the wave-induced pressure term) is more important than the vortex force in the real ocean. Indeed, recent numerical studies show the importance of (the vertical component of) the vortex force associated with the vertical shear of the mean velocity for describing the circulation in surf zones (e.g., Uchiyama et al. 2009, 2010).

\section{The vertical structure of the radiation stress over a sloping bottom}

In the remainder of the manuscript, we focus on the first choice of scaling for the low-pass filtered flow given in section 3. The second choice requires the introduction of viscosity to incorporate the wind stress (cf. Fan et al. 2010) and is not considered further here.

\section{a. First-order waves}

Substitution of (8a)-(8f) and (11a)-(11d) to (1a)-(1d) yields

$$
\begin{aligned}
z_{1 z \tau}^{\prime \prime \prime}+\dot{\mathbf{\nabla}} \cdot \mathbf{V}_{1}^{\prime \prime \prime} & =0, \\
z_{1 \tau}^{\prime \prime \prime} & =w_{1}^{\prime \prime \prime}, \\
\mathbf{V}_{1 \tau}^{\prime \prime \prime} & =-\dot{\nabla}\left(p_{1}^{\prime \prime \prime}+\eta_{1}^{\prime \prime \prime}\right), \\
w_{1 \tau}^{\prime \prime \prime} & =-p_{1 z}^{\prime \prime \prime} .
\end{aligned}
$$

We assume $O(\alpha)$ waves to be monochromatic and (nearly) steady: $\eta_{1}^{\prime \prime \prime}=\mathcal{A} \cos \theta$ where $\mathcal{A}$ is wave amplitude, $\theta=k x+l y-\sigma \tau$ is wave phase with $k$ and $l$ being wavenumbers in the $x$ and $y$ direction, and $\sigma$ is wave frequency. These parameters are constant on the time and spatial scales of waves (i.e., ${ }_{\tau} A=0$ and $\dot{\nabla} A=0$ for $A=\mathcal{A}, k, l, \sigma)$ but may vary on the time and spatial scales of low-pass filtered quantities (i.e., $\partial_{T} A \neq 0$ and $\bar{\nabla} A \neq 0$ for $A=\mathcal{A}, k, l, \sigma)$. With the boundary conditions of $w_{1}^{\prime \prime \prime}=0$ at $z=-h$ [using the assumption that the bottom slope is $O(\alpha)]$ and $p_{1}^{\prime \prime \prime}=0$ at $z=\bar{\eta}$, we solve (16a)-(16d) to yield

$$
\begin{aligned}
\sigma^{2} & =\kappa \tanh \kappa(\bar{\eta}+h), \quad \kappa \equiv \sqrt{k^{2}+l^{2}}, \\
\phi_{1}^{\prime \prime \prime} & =(\mathcal{A} / \kappa) \cos \theta \frac{\cosh \kappa(z+h)}{\sinh \kappa(\bar{\eta}+h)}, \\
\mathbf{V}_{1}^{\prime \prime \prime} & =\dot{\nabla} \phi_{1 \tau}^{\prime \prime \prime}=(-\sigma \dot{\nabla} \theta) \phi_{1 \theta \theta}^{\prime \prime \prime}=\sigma \dot{\nabla} \theta \phi_{1}^{\prime \prime \prime}, \\
w_{1}^{\prime \prime \prime} & =\phi_{1 z \tau}^{\prime \prime \prime}=(-\sigma) \phi_{1 z \theta}^{\prime \prime \prime}, \\
z_{1}^{\prime \prime \prime} & =\phi_{1 z}^{\prime \prime \prime}, \\
p_{1}^{\prime \prime \prime} & =\sigma^{2} \phi_{1}^{\prime \prime \prime}-\eta_{1}^{\prime \prime \prime},
\end{aligned}
$$

where $\dot{\nabla} \theta=(k, l)$ and $\eta_{1}^{\prime \prime \prime}=\left.z_{1}^{\prime \prime \prime}\right|_{z=\bar{\eta}}$ are understood. The above solution is given in the VL coordinates. The wave-induced velocity in Mellor $(2003,2005)$ can be called the quasi-Stokes velocity following McDougall and McIntosh (2001) and can be transformed as follows: ${ }^{5}$

$$
\begin{aligned}
\mathbf{V}_{2}^{q s} & \equiv\left(\overline{z_{1}^{\prime \prime \prime} \mathbf{V}_{1}^{\prime \prime \prime}}\right)_{z}=\left(\overline{\phi_{1 z}^{\prime \prime \prime} \sigma^{2} \phi_{1}^{\prime \prime \prime}}\right)_{z}(\dot{\nabla} \theta) / \sigma \\
& =\left[\overline{z_{1}^{\prime \prime \prime}\left(p_{1}^{\prime \prime \prime}+\eta_{1}^{\prime \prime \prime}\right)}\right]_{z}(\dot{\nabla} \theta) / \sigma .
\end{aligned}
$$

Using (18), the depth integral of the quasi-Stokes velocity can be related with the wave energy,

$$
\int_{-h}^{\bar{\eta}} \mathbf{V}_{2}^{q s} d z=\overline{\left.\eta_{1}^{\prime \prime \prime} \mathbf{V}_{1}^{\prime \prime \prime}\right|_{z=\bar{\eta}}}=\underbrace{\overline{\eta_{1}^{\prime \prime \prime}}}_{E_{2}}(\dot{\mathbf{\nabla}} \theta) / \sigma,
$$

where (6c) and $p_{1}^{\prime \prime \prime}=0$ at $z=\bar{\eta}$ have been used, and $E_{2}=\overline{\eta_{1}^{\prime \prime \prime}}$ is the depth-integrated total (i.e., kinetic plus potential) wave energy at $O\left(\alpha^{2}\right)$. Note that the depth-integrated wave kinetic energy is equal to the wave potential energy:

\footnotetext{
${ }^{5}$ The quasi-Stokes velocity is the extra velocity that must be added to the Eulerian mean velocity to give the total transport velocity [see AG12, their Eqs. (16) and (17)] and is closely related to the Stokes drift, as discussed there.
} 


$$
\begin{aligned}
\frac{1}{2} \int_{-h}^{\bar{\eta}} \overline{\left|\mathbf{V}_{1}^{\prime \prime \prime}\right|^{2}+w_{1}^{\prime \prime \prime}} d z & \left.\left.=\frac{1}{2} \int_{-h}^{\bar{\eta}} \sigma^{2} \overline{\left(\kappa^{2} \phi_{1}^{\prime \prime \prime} 2+\phi_{1 z \theta}^{\prime \prime \prime}\right.}\right) d z=\frac{1}{2} \int_{-h}^{\bar{\eta}} \sigma^{2} \overline{\left(\kappa^{2} \phi_{1}^{\prime \prime \prime}+\phi_{1 z}^{\prime \prime 2}\right.}\right) d z \\
& =\frac{1}{2} \int_{-h}^{\bar{\eta}} \sigma^{2}\left(\overline{\phi_{1}^{\prime \prime \prime} \phi_{1 z}^{\prime \prime \prime}}\right)_{z} d z=\frac{1}{2} \int_{-h}^{\bar{\eta}}\left[\overline{\left(p_{1}^{\prime \prime \prime}+\eta_{1}^{\prime \prime \prime}\right) z_{1}^{\prime \prime \prime}}\right]_{z} d z=\frac{1}{2} \overline{\eta_{1}^{\prime \prime \prime}}
\end{aligned}
$$

where (17c)-(17f) have been used.

The depth-integrated radiation stress term is given by the difference of (7a) and (7b)

$$
\begin{aligned}
& \int_{-h}^{\bar{\eta}}\left[\mathcal{R} \mathcal{S}_{3}^{\mathbf{V}}-\mathcal{F} \mathcal{S}_{3}^{\mathbf{V}}\right] d z=\overline{\boldsymbol{\nabla}} \cdot \int_{-h}^{\bar{\eta}} \overline{\mathbf{V}_{1}^{\prime \prime \prime} \mathbf{V}_{1}^{\prime \prime \prime}} d z+\frac{1}{2} \overline{\bar{\nabla}} \overline{\eta_{1}^{\prime \prime \prime}}-\overline{\boldsymbol{\nabla}} \int_{-h}^{\bar{\eta}}\left(\overline{z_{1}^{\prime \prime \prime} p_{1 z}^{\prime \prime \prime}}\right) d z \\
& =\bar{\nabla} \cdot \int_{-h}^{\bar{\eta}} \overline{\mathbf{V}_{1}^{\prime \prime \prime} \mathbf{V}_{1}^{\prime \prime \prime}} d z+\frac{1}{2} \bar{\nabla} \int_{-h}^{\bar{\eta}} \overline{\left|\mathbf{V}_{1}^{\prime \prime \prime}\right|^{2}+w_{1}^{\prime \prime \prime}} d z+\bar{\nabla} \int_{-h}^{\bar{\eta}}\left(\overline{z_{1}^{\prime \prime \prime} w_{1 \tau}^{\prime \prime \prime}}\right) d z \\
& =\bar{\nabla} \cdot \int_{-h}^{\bar{\eta}} \overline{\mathbf{V}_{1}^{\prime \prime \prime} \mathbf{V}_{1}^{\prime \prime \prime}} d z+\bar{\nabla} \int_{-h}^{\bar{\eta}} J d z=\overline{\mathbf{\nabla}} \cdot \int_{-h}^{\bar{\eta}} \overline{\mathbf{V}_{1}^{\prime \prime \prime}\left(p_{1}^{\prime \prime \prime}+\eta_{1}^{\prime \prime \prime}\right)}(\dot{\mathbf{\nabla}} \theta) / \sigma d z+\overline{\boldsymbol{\nabla}} \int_{-h}^{\bar{\eta}} J d z \\
& =\bar{\nabla} \cdot\left[\mathbf{C}_{g} E_{2}(\dot{\nabla} \theta) / \sigma\right]+\bar{\nabla} \int_{-h}^{\bar{\eta}} J d z=\bar{\nabla} \cdot\left(\mathbf{C}_{g} \overline{\left.\eta_{1}^{\prime \prime \prime} \mathbf{V}_{1}^{\prime \prime \prime}\right|_{z=\bar{\eta}}}\right)+\bar{\nabla} \int_{-h}^{\bar{\eta}} J d z,
\end{aligned}
$$

where the second line has been derived using (20), the third line has been derived using (16b), and the last line has been derived using (19). The symbol $J$ comes from Smith (2006) and is defined by

$$
J \equiv \frac{1}{2}\left(\overline{\left|\mathbf{V}_{1}^{\prime \prime \prime}\right|^{2}-w_{1}^{\prime \prime \prime}}\right),
$$

which turns out to be a depth-independent quantity because $\cosh ^{2}-\sinh ^{2}=1$. The symbol $\mathbf{C}_{g}=\partial \sigma / \partial(k, l)$ is the group velocity in classical linear wave theory. Also, $J$ is the same as the wave-induced pressure term given by Eq. (39) on page 45 of ARB08. To summarize, the depth-integrated radiation stress term is available using only the first-order wave solution even if the bottom is sloped. However the depth-dependent radiation stress term, $\mathcal{R S}_{3}^{\mathbf{V}}-\mathcal{F S}_{3}^{\mathbf{V}}$ based on (10a)-(10b), requires the second-order wave solution (Ardhuin et al. 2008a, section 4).

\section{b. Second-order waves associated with nonlinear terms}

We decompose the solution of the second-order waves into that associated with the nonlinear terms of (1a)-(1d) and that associated with the bottom slope. The equation system for the former solution is derived from (1a)-(1d):

$$
\begin{gathered}
z_{2 z \tau}^{\prime \prime \prime}+\dot{\mathbf{V}} \cdot\left(\mathbf{V}_{2}^{\prime \prime \prime}+z_{1 z}^{\prime \prime \prime} \mathbf{V}_{1}^{\prime \prime \prime}\right)=0, \\
z_{2 \tau}^{\prime \prime \prime}+\mathbf{V}_{1}^{\prime \prime \prime} \cdot \dot{\nabla} z_{1}^{\prime \prime \prime}=w_{2}^{\prime \prime \prime}, \\
\mathbf{V}_{2 \tau}^{\prime \prime \prime}+\mathbf{V}_{1}^{\prime \prime \prime} \cdot \dot{\mathbf{V}} \mathbf{V}_{1}^{\prime \prime \prime}=-\dot{\nabla}\left(p_{2}^{\prime \prime \prime}+\eta_{2}^{\prime \prime \prime}\right)-w_{1 \tau}^{\prime \prime \prime} \dot{\nabla} z_{1}^{\prime \prime \prime}, \\
w_{2 \tau}^{\prime \prime \prime}+\mathbf{V}_{1}^{\prime \prime \prime} \cdot \dot{\nabla} w_{1}^{\prime \prime \prime}=-p_{2 z}^{\prime \prime \prime}-w_{1 \tau}^{\prime \prime \prime} z_{1 z}^{\prime \prime \prime}
\end{gathered}
$$

which can be solved using the boundary conditions of $\left.w_{2}^{\prime \prime \prime}\right|_{z=-h}=0$ and $\left.p_{2}^{\prime \prime \prime}\right|_{z=\bar{\eta}}=0$ (appendix B). The solution is proportional to $\cos 2 \theta$ (or $\sin 2 \theta$ ) with the consequence that it does not correlate with the first-order solution in the calculation of the Reynolds stress term (10a) and the form stress term (10b). ${ }^{6}$

\section{c. Second-order waves associated with the bottom slope}

The equation system for the solution associated with the bottom slope is based on the linear terms of (1a)-(1d),

$$
\begin{gathered}
\left(z_{1 T}^{\prime \prime \prime}+z_{2 \tau}^{\prime \prime \prime}+w_{2}^{* \prime \prime}\right)_{z}+\bar{\nabla} \cdot \mathbf{V}_{1}^{\prime \prime \prime}+\dot{\mathbf{\nabla}} \cdot \mathbf{V}_{2}^{\prime \prime \prime}=0 \\
z_{1 T}^{\prime \prime \prime}+z_{2 \tau}^{\prime \prime \prime}+w_{2}^{* \prime \prime \prime}=w_{2}^{\prime \prime \prime}, \\
\mathbf{V}_{1 T}^{\prime \prime \prime}+\mathbf{V}_{2 \tau}^{\prime \prime \prime}=-\dot{\nabla}\left(p_{2}^{\prime \prime \prime}+\eta_{2}^{\prime \prime \prime}\right)-\bar{\nabla}\left(p_{1}^{\prime \prime \prime}+\eta_{1}^{\prime \prime \prime}\right), \\
w_{1 T}^{\prime \prime \prime}+w_{2 \tau}^{\prime \prime \prime}=-p_{2 z}^{\prime \prime \prime},
\end{gathered}
$$

which can be solved using the boundary conditions of $\left.w_{2}^{\prime \prime \prime}\right|_{z=-h}=-\left.\mathbf{V}_{1}^{\prime \prime \prime}\right|_{z=-h} \cdot \bar{\nabla} h$ and $\left.p_{2}^{\prime \prime \prime}\right|_{z=\bar{\eta}}=0$. The exact solution in the Eulerian coordinates has been given by Chu and Mei (1970) and Zou et al. (2003, see their appendix). It would be possible to derive the corresponding solution using the VL coordinates. However it would be more useful if the depth-dependent form stress term (10b) can be transformed into an expression where the second-order wave solution does not appear. This is shown in what follows using the recipe of AGVF.

\footnotetext{
${ }^{6}$ When nonmonochromatic waves are considered, the solution is more complicated and second-order waves arising from the nonlinear terms cannot be neglected as in the present study.
} 
The equation system (16a)-(16d) and (24a)-(24d) may be written in a general form,

$$
\begin{aligned}
z_{z t}^{\prime \prime \prime} & =-\nabla \cdot \mathbf{V}^{\prime \prime \prime}-\left(w^{* \prime \prime \prime}\right)_{z}, \\
z_{t}^{\prime \prime \prime} & =w^{\prime \prime \prime}-w^{* \prime \prime \prime}, \\
\mathbf{V}_{t}^{\prime \prime \prime} & =-\nabla\left(p^{\prime \prime \prime}+\eta^{\prime \prime \prime}\right), \\
w_{t}^{\prime \prime \prime} & =-p_{z}^{\prime \prime \prime}
\end{aligned}
$$

where $\partial_{t}=\partial_{\tau}+\alpha \partial_{T}, \nabla=\dot{\nabla}+\alpha \bar{\nabla}$, and $A^{\prime \prime \prime}=\alpha A_{1}^{\prime \prime \prime}+$ $\alpha^{2} A_{2}^{\prime \prime \prime}$ for an arbitrary quantity $A^{\prime \prime \prime}$. Using (25c)-(25d), the form stress term $(10 \mathrm{~b})$ can be rewritten

$$
\begin{aligned}
\mathcal{F} \mathcal{S}^{\mathbf{V}} & =-\overline{z_{z}^{\prime \prime \prime} \mathbf{\nabla}\left(p^{\prime \prime \prime}+\eta^{\prime \prime \prime}\right)}+\overline{\left(\nabla z^{\prime \prime \prime}\right) p_{z}^{\prime \prime \prime}} \\
& =\overline{z_{z}^{\prime \prime \prime} \mathbf{V}_{t}^{\prime \prime \prime}}-\overline{\left(\nabla z^{\prime \prime \prime}\right) w_{t}^{\prime \prime \prime}} .
\end{aligned}
$$

Using (25a), the first term on the last line of (26) can be rewritten:

$$
\begin{aligned}
& \overline{z_{z}^{\prime \prime \prime} \mathbf{V}_{t}^{\prime \prime \prime}}=\left(\overline{z_{z}^{\prime \prime \prime} \mathbf{V}^{\prime \prime \prime}}\right)_{t}-\overline{z_{z t}^{\prime \prime \prime} \mathbf{V}^{\prime \prime \prime}}=\left(\overline{z_{z}^{\prime \prime \prime} \mathbf{V}^{\prime \prime \prime}}\right)_{t}+\overline{\left(\boldsymbol{\nabla} \cdot \mathbf{V}^{\prime \prime \prime}+w_{z}^{* \prime \prime}\right) \mathbf{V}^{\prime \prime \prime}} \\
& =\left(\overline{z_{z}^{\prime \prime \prime} \mathbf{V}^{\prime \prime \prime}}\right)_{t}+\overline{\left[\boldsymbol{\nabla} \cdot\left(\mathbf{V}^{\prime \prime \prime} \mathbf{V}^{\prime \prime \prime}\right)+\left(w^{* \prime \prime \prime} \mathbf{V}^{\prime \prime \prime}\right)_{z}\right]}-\overline{\mathbf{V}^{\prime \prime \prime} \cdot \nabla \mathbf{V}^{\prime \prime \prime}}-\overline{w^{* \prime \prime \prime} \mathbf{V}_{z}^{\prime \prime \prime}} \\
& =\left(\overline{z_{z}^{\prime \prime \prime} \mathbf{V}^{\prime \prime \prime}}\right)_{t}+\mathcal{R} \mathcal{S}^{\mathbf{V}}-\overline{\mathbf{V}^{\prime \prime \prime} \cdot \nabla \mathbf{V}^{\prime \prime \prime}}-\overline{w^{* \prime \prime \prime} \mathbf{V}_{z}^{\prime \prime \prime}} \\
& =\left(\overline{z_{z}^{\prime \prime \prime} \mathbf{V}^{\prime \prime \prime}}\right)_{t}+\mathcal{R} \mathcal{S}^{\mathbf{V}}-\frac{1}{2} \nabla \mid \overline{\left.\mathbf{V}^{\prime \prime \prime}\right|^{2}}-\overline{\left(\mathbf{\nabla} \times \mathbf{V}^{\prime \prime \prime}\right) \times \mathbf{V}^{\prime \prime \prime}}-\overline{w^{* \prime \prime \prime} \mathbf{V}_{z}^{\prime \prime \prime}} .
\end{aligned}
$$

Using (25b), the second term on the last line of (26) can be rewritten:

$$
\begin{aligned}
-\overline{\left(\nabla z^{\prime \prime \prime}\right) w_{t}^{\prime \prime \prime}} & \left.=-\overline{\left[\left(\nabla z^{\prime \prime \prime}\right) w^{\prime \prime \prime}\right.}\right]_{t}+\overline{\left(\nabla z_{t}^{\prime \prime \prime}\right) w^{\prime \prime \prime}} \\
& \left.=-\overline{\left[\left(\nabla z^{\prime \prime \prime}\right) w^{\prime \prime \prime}\right.}\right]_{t}+\overline{\left(\nabla w^{\prime \prime \prime}-\nabla w^{* \prime \prime}\right) w^{\prime \prime \prime}} \\
& \left.=-\overline{\left[\left(\nabla z^{\prime \prime \prime}\right) w^{\prime \prime \prime}\right.}\right]_{t}+\nabla\left(\frac{1}{2} \overline{w^{\prime \prime \prime}}-\overline{w^{\prime \prime \prime} w^{* \prime \prime \prime}}\right)+\overline{\left(\nabla w^{\prime \prime \prime}\right) w^{* \prime \prime \prime}}
\end{aligned}
$$

We substitute (27a)-(27b) to (26) then pick-up terms at $O\left(\alpha^{3}\right)$ to yield

$$
\begin{aligned}
\mathcal{F} \mathcal{S}_{3}^{\mathbf{V}=} & {\left[\overline{z_{1 z}^{\prime \prime \prime} \mathbf{V}_{1}^{\prime \prime \prime}-\left(\dot{\nabla} z_{1}^{\prime \prime \prime}\right) w_{1}^{\prime \prime \prime}}\right]_{T}+\mathcal{R} \mathcal{S}_{3}^{\mathbf{V}} } \\
& -\frac{1}{2} \bar{\nabla}\left(\overline{\left|\mathbf{V}_{1}^{\prime \prime \prime}\right|^{2}-w_{1}^{\prime \prime \prime}}\right)-\left[\overline{\left(\bar{\nabla} \times \mathbf{V}^{\prime \prime \prime}\right) \times \mathbf{V}^{\prime \prime \prime}}\right]_{3},
\end{aligned}
$$

where $w_{1}^{* \prime \prime \prime}=0$ and $\mathbf{V}_{1 z}^{\prime \prime \prime}-\dot{\nabla} w_{1}^{\prime \prime \prime}=0$ have been used.

The first term on the rhs of (28) is the temporal derivative of "the pseudomomentum in the VL framework"
(AGVF) and can be transformed to the quasi-Stokes velocity,

$$
\begin{aligned}
\overline{z_{1 z}^{\prime \prime \prime} \mathbf{V}_{1}^{\prime \prime \prime}-\left(\dot{\nabla} z_{1}^{\prime \prime \prime}\right) w_{1}^{\prime \prime \prime}} & =\overline{z_{1 z}^{\prime \prime \prime} \mathbf{V}_{1}^{\prime \prime \prime}+z_{1}^{\prime \prime \prime} \dot{\nabla} w_{1}^{\prime \prime \prime}} \\
& =\overline{z_{1 z}^{\prime \prime \prime} \mathbf{V}_{1}^{\prime \prime \prime}+z_{1}^{\prime \prime \prime} \mathbf{V}_{1 z}^{\prime \prime \prime}}=\left(\overline{z_{1}^{\prime \prime \prime} \mathbf{V}_{1}^{\prime \prime \prime}}\right)_{z} .
\end{aligned}
$$

Namely, as long as the $O(\alpha)$ wave is horizontally homogeneous and irrotational in the vertical plane, the analytical expression of the VL pseudomomentum is identical to the quasi-Stokes velocity. Because $O(\alpha)$ waves satisfy $\dot{\boldsymbol{\nabla}} \times \mathbf{V}_{1}^{\prime \prime \prime}=0$, the last term of (28) may be rewritten

$$
\begin{aligned}
& {\left[\overline{\left(\boldsymbol{\nabla} \times \mathbf{V}^{\prime \prime \prime}\right) \times \mathbf{V}^{\prime \prime \prime}}\right]_{3}=\overline{\left(\bar{\nabla} \times \mathbf{V}_{1}^{\prime \prime \prime}+\dot{\mathbf{V}} \times \mathbf{V}_{2}^{\prime \prime \prime}\right) \times \mathbf{V}_{1}^{\prime \prime \prime}}=-\overline{\left(\bar{\nabla} \times \mathbf{V}_{1 \tau}^{\prime \prime \prime}+\dot{\boldsymbol{\nabla}} \times \mathbf{V}_{2 \tau}^{\prime \prime \prime}\right) \times \dot{\boldsymbol{\nabla}} \phi_{1}^{\prime \prime \prime}}} \\
& =\overline{\left[\bar{\nabla} \times \dot{\nabla}\left(p_{1}^{\prime \prime \prime}+\eta_{1}^{\prime \prime \prime}\right)+\dot{\nabla} \times \bar{\nabla}\left(p_{1}^{\prime \prime \prime}+\eta_{1}^{\prime \prime \prime}\right)\right] \times \dot{\nabla} \phi_{1}^{\prime \prime \prime}} \\
& =\overline{\left[\overline{\mathbf{\nabla}} \times\left(\sigma^{2} \phi_{1 \theta}^{\prime \prime \prime} \dot{\nabla} \theta\right)+(\dot{\mathbf{\nabla}} \theta) \times \overline{\mathbf{\nabla}}\left(\sigma^{2} \phi_{1 \theta}^{\prime \prime \prime}\right)\right] \times \dot{\mathbf{\nabla}} \phi_{1}^{\prime \prime \prime}}=\overline{\left[\sigma^{2} \phi_{1 \theta}^{\prime \prime \prime}(\overline{\mathbf{\nabla}} \times \dot{\mathbf{\nabla}} \theta)\right] \times \dot{\mathbf{\nabla}} \phi_{1}^{\prime \prime \prime}}=0,
\end{aligned}
$$

where $(17 \mathrm{c})$ has been used to derive the first line, and both (16c) and (24c) have been used to derive the second line. The procedure through (26)-(30) is based on AGVF.

\section{d. Depth-dependent radiation stress}

Substitution of (22) and (29)-(30) to (28) yields the depth-dependent radiation stress term applicable to circulation on a sloping bottom, 
(a) GLM
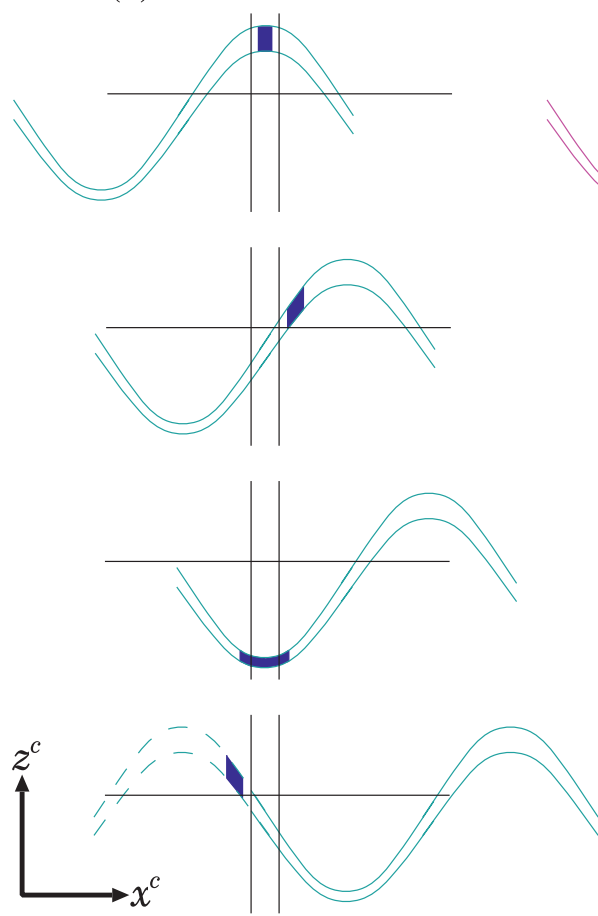

(b) VL
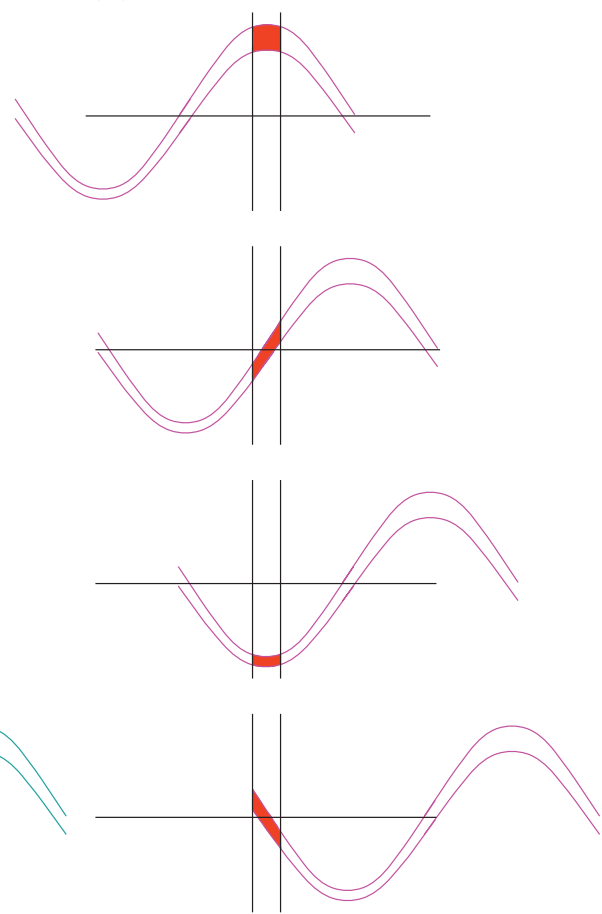

FIG. 2. Illustration of the phase cycle of a wave propagating in the direction of $x^{c}$ axis. A control volume element in (a) the generalized-Lagrangian-mean (GLM) coordinates of Andrews and McIntyre (1978) and (b) the vertically Lagrangian (VL) coordinates of the present study is shaded in blue and red, respectively, with its low-pass-filtered height, as measured in each coordinate system, being indicated by horizontal lines, and the reference horizontal position being indicated by vertical lines. Each color line indicates a material surface which is formed by connecting the instantaneous position of water particles whose threedimensionally Lagrangian low-pass-filtered height is a given value.

$$
\mathcal{R S}_{3}^{\mathbf{V}}-\mathcal{F} \mathcal{S}_{3}^{\mathbf{V}}=-\left(\overline{z_{1}^{\prime \prime \prime} \mathbf{V}_{1}^{\prime \prime \prime}}\right)_{z T}+\bar{\nabla} J
$$

which contains no singular treatment at the sea surface, in contrast to Mellor (2008, 2011a,b). Moreover (31) does not require the solution of the second-order waves. Substitution of (31) to (12b) yields

$$
\partial_{T}\left[\hat{\mathbf{V}}_{2}-\left(\overline{z_{1}^{\prime \prime \prime} \mathbf{V}_{1}^{\prime \prime \prime}}\right)_{z}\right]=-\bar{\nabla}(\underbrace{\bar{p}_{2}}_{0}+\bar{\eta}_{2}+J) .
$$

The left-hand side is the time development of $\hat{\mathbf{V}}_{2}-$ $\left(\overline{z_{1}^{\prime \prime \prime} \mathbf{V}_{1}^{\prime \prime \prime}}\right)_{z}=\overline{\mathbf{V}}_{2}-\overline{z_{1}^{\prime \prime \prime} \mathbf{V}_{1 z}^{\prime \prime \prime}} \equiv \overline{\mathbf{V}}_{2}^{c}$, where the last term is the Eulerian mean velocity. Namely the wave-averaged momentum equation (32) has been written for the development of the Eulerian mean velocity, which is as in the three-dimensional Lagrangian mean framework of AM78. The unweighted mean nonhydrostatic pressure $\bar{p}_{2}$ in (32) is zero owing to (12c) and because we can put $\bar{p}_{2}=0$ at the surface (since we are not taking account of variations in atmospheric pressure in either space or time).
In contrast to Smith (2006), who used depth-integrated equations, the $J$ term on the rhs of (32) has been derived from depth-dependent equations in the present study. Our simple derivation in section $4 \mathrm{c}$ is complementary to both Lane et al. (2007) who identified the $J$ term (see $\hat{\zeta}$ on page 1127 of their paper) in the three-dimensional Eulerian mean framework of MRL04 yet with a separate treatment of the vicinity of the sea surface, and ARB08 (see $S^{J}$ on page 45 of their paper) who presented a rigorous derivation using the three-dimensional Lagrangian mean framework. The VL coordinate system has the advantage that no special treatment is required near the sea surface, in contrast to the three-dimensional Eulerian mean framework, and there is no misalignment of the mean surface height, in contrast to the three-dimensional Lagrangian mean framework [see Fig. 2 and McIntyre (1988)]. Although our approach in section $4 \mathrm{c}$ is simple and recommended, it is left

\footnotetext{
${ }^{7}$ This concern has been eliminated in ARB08 at least to the leading order in terms of a perturbation expansion.
} 
for a future study to rederive (31) using the approach of Garrett (1976) and Smith (2006) so as to see how to apply the set of the wave energy equation and the wave crest equation to the depth-dependent problem (appendix C).

It is of interest to compare the unweighted-mean nonhydrostatic pressure $\bar{p}_{2}(=0)$ (averaged in the VL coordinates) with the Eulerian mean nonhydrostatic pressure $\bar{p}_{2}^{c}$ (averaged in Eulerian-Cartesian coordinates) using a Taylor expansion in the vertical direction (appendix D) to read

$$
\begin{aligned}
\bar{p}_{2}^{c} & \equiv \bar{p}_{2}-\overline{z_{1}^{\prime \prime \prime} p_{1 z}^{\prime \prime \prime}}=\bar{p}_{2}+\overline{z_{1}^{\prime \prime \prime} w_{1 \tau}^{\prime \prime \prime}}=\bar{p}_{2}-\overline{z_{1 \tau}^{\prime \prime \prime} w_{1}^{\prime \prime \prime}} \\
& =\bar{p}_{2}-\overline{w_{1}^{\prime \prime \prime}}=-\overline{w_{1}^{\prime \prime \prime}}
\end{aligned}
$$

where (17d)-(17f) have been used. Substitution of $(0=) \bar{p}_{2}=\bar{p}_{2}^{c}+\overline{w_{1}^{\prime \prime \prime}}$ to the rhs of (32) yields

$$
\partial_{T} \overline{\mathbf{V}}_{2}^{c}=-\bar{\nabla}[\underbrace{\bar{p}_{2}^{c}}_{-\overline{w_{1}^{\prime \prime \prime}}}+\bar{\eta}_{2}+\frac{1}{2}\left(\mid \overline{\left.\mathbf{V}_{1}^{\prime \prime \prime}\right|^{2}+w_{1}^{\prime \prime \prime}}\right)],
$$

which is similar to the Eulerian average of the vectorinvariant form of momentum equations with (the traditional form of) the Bernoulli head and no vorticity (not shown). To summarize, the $J$ term can be interpreted as the sum of (the traditional form of) the Bernoulli head and the Eulerian mean nonhydrostatic pressure $\bar{p}^{c}$ (Smith 2006, section 5).

\section{The connection to previous studies}

\section{a. Smith (2006) and LHS64}

The depth-integral of (31) is

$$
\begin{aligned}
\int_{-h}^{\bar{\eta}} & {\left[\mathcal{R} \mathcal{S}_{3}^{\mathbf{V}}-\mathcal{F} \mathcal{S}_{3}^{\mathbf{V}}\right] d z } \\
& =-\left(\overline{\left.\eta_{1}^{\prime \prime \prime} \mathbf{V}_{1}^{\prime \prime \prime}\right|_{z=\bar{\eta}}}\right)_{T}+\bar{\nabla} \int_{-h}^{\bar{\eta}} J d z-\left.J\right|_{z=-h} \bar{\nabla} h .
\end{aligned}
$$

The difference of (21) and (35) yields

$\left(\overline{\left.\eta_{1}^{\prime \prime \prime} \mathbf{V}_{1}^{\prime \prime \prime}\right|_{z=\bar{\eta}}}\right)_{T}+\bar{\nabla} \cdot\left(\mathbf{C}_{g} \overline{\left.\eta_{1}^{\prime \prime \prime} \mathbf{V}_{1}^{\prime \prime \prime}\right|_{z=\bar{\eta}}}\right)=-J \bar{\nabla} h$,

which is identical to (2.27) of Smith (2006).

To see the connection with LHS64, we return to (21). We rewrite (21) as follows:

$$
\begin{aligned}
& \int_{-h}^{\bar{\eta}}\left[\mathcal{R S}_{3}^{\mathbf{V}}-\mathcal{F} \mathcal{S}_{3}^{\mathbf{V}}\right] d z=\bar{\nabla} \cdot \int_{=h}^{\bar{\eta}} \overline{\mathbf{V}_{1}^{\prime \prime \prime} \mathbf{V}_{1}^{\prime \prime \prime}} d z+\frac{1}{2} \bar{\nabla} \overline{\eta_{1}^{\prime \prime \prime}}-\bar{\nabla} \int_{=h}^{\bar{\eta}}\left(\overline{z_{1}^{\prime \prime \prime} p_{1 z}^{\prime \prime \prime}}\right) d z \\
& =\bar{\nabla} \cdot \int_{-h}^{\bar{\eta}} \overline{\mathbf{V}_{1}^{\prime \prime \prime} \mathbf{V}_{1}^{\prime \prime \prime}} d z+\frac{1}{2} \bar{\nabla} \overline{\eta_{1}^{\prime \prime \prime}}+\bar{\nabla} \int_{-h}^{\bar{\eta}}\left(\overline{z_{1}^{\prime \prime \prime} w_{1 \tau}^{\prime \prime \prime}}\right) d z \\
& =\bar{\nabla} \cdot \underbrace{\int_{-h}^{\bar{\eta}} \overline{\mathbf{V}_{1}^{\prime \prime \prime} \mathbf{V}_{1}^{\prime \prime \prime}} d z}_{S_{x x}^{(1)}}+\bar{\nabla} \underbrace{\left.\int_{-h}^{\bar{\eta}} \overline{\left(-w_{1}^{\prime \prime \prime}\right.}\right) d z}_{S_{x x}^{(2)}}+\underbrace{\bar{\nabla}}_{S_{x x}^{(3)}} \overline{\frac{\eta_{1}^{\prime \prime 2}}{2}},
\end{aligned}
$$

where $S_{x x}^{(1)}, S_{x x}^{(2)}$, and $S_{x x}^{(3)}$ are the notation in LHS64. Contrary to Mellor $(2008,2011 \mathrm{a}, \mathrm{b})$, we have reproduced the result from LHS64 without recourse to introducing a delta function at the mean sea surface height in the expression for the depth-dependent radiation stress, that is (31).

\section{b. Mellor (2003)}

The sea surface height $\eta$ in (1c) originates from the explicit treatment of hydrostatic pressure in AG12. The connection to the implicit treatment of hydrostatic pressure in papers by Mellor is as follows. The rhs of (1c) may be written as

$$
\begin{aligned}
-\nabla(p+\eta)+p_{z^{c}} \nabla z^{c} & =-\nabla^{c}(p+\eta)=-\nabla^{c}\left[p+\left(\eta-z^{c}\right)\right] \\
& =-\nabla \underbrace{\left[p+\left(\eta-z^{c}\right)\right]}_{p^{\mathrm{Mel}}}+\underbrace{\left(p_{z^{c}}-1\right)}_{\left(p^{\mathrm{Mel}}\right)_{z^{c}}} \nabla z^{c},
\end{aligned}
$$

where $\nabla^{c} z^{c}=0$ has been used to derive the first line, and the symbol $p^{\mathrm{Mel}} \equiv p+\left(\eta-z^{c}\right)$ is the (combined hydrostatic and nonhydrostatic) pressure in Mellor (2003, 2005, 2008, 2011a,b). The TWM average of the rhs of (38) leads to the form stress term and is given by

$$
\begin{aligned}
& -\overline{z_{z}^{c} \nabla\left(p^{\mathrm{Mel}}\right)}+\overline{\left(p^{\mathrm{Mel}}\right)_{z} \nabla z^{c}} \\
& =-\nabla\left(\overline{p^{\mathrm{Mel}}}\right)-\overline{z_{z}^{\prime \prime \prime} \nabla\left(p^{\mathrm{Mel}}\right)^{\prime \prime \prime}}+\overline{\left(p^{\mathrm{Mel}}\right)_{z}^{\prime \prime \prime} \nabla z^{\prime \prime \prime}} \\
& =-\nabla\left(\overline{p^{\mathrm{Mel}}}\right)-\nabla\left[\overline{\underbrace{\left(p^{\mathrm{Mel}}\right)^{\prime \prime \prime} z_{z}^{\prime \prime \prime}}_{\text {Eq. (34e) of M03 }}}\right]+[\underbrace{\overline{\left(p^{\mathrm{Mel}}\right)^{\prime \prime \prime} \nabla z^{\prime \prime \prime}}}_{\text {Eq. (34f) of M03 }}]_{z}
\end{aligned}
$$

where the second and third terms on the last line correspond to Eqs. (34e) and (34f) of Mellor (2003), respectively. We write the negative of these terms at $O\left(\alpha^{3}\right)$, 


$$
\begin{aligned}
& \bar{\nabla}\left[\overline{\left(p^{\mathrm{Mel}}\right)^{\prime \prime \prime} z_{z}^{\prime \prime \prime}}\right]_{2}=\bar{\nabla}\left[\overline{\left(p_{1}^{\prime \prime \prime}+\eta_{1}^{\prime \prime \prime}\right) z_{1}^{\prime \prime \prime}}\right]-\bar{\nabla}\left(\overline{z_{1}^{\prime \prime \prime} z_{1 z}^{\prime \prime \prime}}\right) \\
& =\bar{\nabla}\left[\overline{\left(\sigma^{2} \phi_{1}^{\prime \prime \prime}\right) \phi_{1 z z}^{\prime \prime \prime}}\right]-\bar{\nabla}\left(\overline{z_{1}^{\prime \prime \prime} z_{1 z}^{\prime \prime \prime}}\right) \\
& =\bar{\nabla}\left[\overline{\left(\sigma^{2} \phi_{1}^{\prime \prime \prime}\right) \kappa^{2} \phi_{1}^{\prime \prime \prime}}\right]-\bar{\nabla}\left(\overline{z_{1}^{\prime \prime \prime} z_{1 z}^{\prime \prime \prime}}\right) \\
& =\overline{\mathbf{\nabla}} \overline{\left.\mathbf{V}_{1}^{\prime \prime \prime}\right|^{2}}-\bar{\nabla}\left(\overline{z_{1}^{\prime \prime \prime} z_{1 z}^{\prime \prime \prime}}\right) \\
& =-\bar{\nabla} \overline{w_{1}^{\prime \prime \prime}}+\bar{\nabla}\left(\mid \overline{\left.\mathbf{V}_{1}^{\prime \prime \prime}\right|^{2}+w_{1}^{\prime \prime \prime}}\right)-\bar{\nabla}\left(\overline{z_{1}^{\prime \prime \prime} z_{1 z}^{\prime \prime \prime}}\right) \text {, }
\end{aligned}
$$

and

$$
\begin{aligned}
-\left[\overline{\left(p^{\mathrm{Mel}}\right)^{\prime \prime \prime} \nabla z^{\prime \prime \prime}}\right]_{3 z}= & -\left[\overline{\left(p_{1}^{\prime \prime \prime}+\eta_{1}^{\prime \prime \prime}-z_{1}^{\prime \prime \prime}\right) \bar{\nabla} z_{1}^{\prime \prime \prime}}\right]_{z} \\
& -\left[\overline{\left(p_{1}^{\prime \prime \prime}+\eta_{1}^{\prime \prime \prime}-z_{1}^{\prime \prime \prime}\right) \dot{\nabla} z_{2}^{\prime \prime \prime}}\right]_{z} \\
& -\left[\overline{\left(p_{2}^{\prime \prime \prime}+\eta_{2}^{\prime \prime \prime}-z_{2}^{\prime \prime \prime}\right) \dot{\nabla} z_{1}^{\prime \prime \prime}}\right]_{z} .
\end{aligned}
$$

We consider the depth integral of (40a)-(40b). As stated in footnote 2 of this manuscript, the choice of the flux divergence as expressed by the last two terms of (39) is (correct but) inconvenient for handling the sloping bottom boundary condition, in particular when taking the depth integral. Hence we must assume the bottom is flat [or the slope is at most $O\left(\alpha^{2}\right)$ ]. The depth integral of the first term of the last line of (40a) becomes $S_{x x}^{(2)}$ in (37). The depth integral of the sum of the last two terms of (40a) and the use of (20) yield $S_{x x}^{(3)}$ in (37). The depth integral of the rhs of (40b) vanishes. Including the Reynolds stress term then recovers the expression for the vertically integrated radiation stress from LHS64, as in (37).

It is noteworthy that we have related our analysis to Eqs. (34e) and (34f) in Mellor (2003). Mellor (2011b) recommends that Eq. (34e) be deleted from Mellor (2003) and argues that Eq. (34f) is identically zero. Clearly we do not support these recommendations. Although Eq. (34f) in Mellor (2003) is zero after vertical integration, as stated in Mellor (2003), is not necessarily zero at all depths. This is because of the slow spatial derivative operator in the first term on the rhs of (40b).

\section{c. Mellor (2008, 2011a)}

In Mellor $(2008,2011 a)$, the pressure terms are treated using Eulerian averaging. Mellor then considered the depth integral of (the combined nonhydrostatic and hydrostatic) pressure. He computed the difference of $\overline{\int_{-h}^{\eta} p^{\mathrm{Mel}} d z^{c}}$ and $\int_{-h}^{\bar{\eta}} \overline{p^{\mathrm{Mel}}{ }^{c}} d z$ to yield $\overline{\eta^{\prime \prime \prime}} / 2$. When determining the vertical profile of the wave-averaged pressure, he introduced the delta function at the height of the mean sea surface to account for the difference of $\overline{\eta^{\prime \prime \prime}} / 2$.

To gain further insight we derive the following general expression for the time average of the depth integral of an arbitrary quantity $A$ in terms of TWM and Eulerian mean quantities:

$$
\begin{aligned}
\overline{\int_{-h}^{\eta} A d z^{c}} & =\overline{\int_{-h}^{\bar{\eta}} A z_{z}^{c} d z}=\int_{-h}^{\bar{\eta}} \overline{A z_{z}^{c}} d z=\int_{-h}^{\bar{\eta}} \hat{A} d z \\
& =\int_{-h}^{\bar{\eta}} \bar{A}^{c} d z+\left[\left.\overline{\eta^{\prime \prime \prime} A^{\prime \prime \prime}}\right|_{z=\bar{\eta}}-\left.\left(\overline{\eta^{\prime \prime \prime}} / 2\right) \bar{A}_{z}\right|_{z=\bar{\eta}}\right],
\end{aligned}
$$

where the last line has been derived using (D2) in appendix D. Substitution of $A=p^{\mathrm{Mel}}\left(\equiv p+\eta-z^{c}\right)$ to the second term on the last line of (41) [and the use of $\left(p^{\mathrm{Mel}}\right)^{\prime \prime \prime}=0$ and $\left(\overline{p^{\mathrm{Mel}}}\right)_{z}=\overline{p_{z}-z_{z}^{c}}=-1$ at $\left.z=\bar{\eta}\right]$ yields $\overline{\eta^{\prime \prime \prime}} / 2$, which is consistent with Mellor (2008, 2011a). However this term originates from the second term on the last line of (D2) which actually has a continuous vertical profile even if $A=p^{\mathrm{Mel}}$.

\section{Summary}

We have derived the depth-dependent radiation stress term for the effect of surface gravity waves on circulations on a sloping bottom. The derivation has been carried out using the thickness weighted mean (TWM) equations in the vertically Lagrangian (VL) coordinates of AG12 in which the radiation stress corresponds to the Reynolds stress minus the form stress. A feature of our analysis is the consistent use of a perturbation expansion for both waves and circulation in formulating the equations. The fact that we focus on the scaling associated with LHS64 is for the purpose of mathematical clarity regarding the attempt of Mellor $(2003,2008)$ to derive a depth-dependent version of LHS64, and does not necessarily indicate that the radiation stress (or the wave-induced pressure term) is more important than the vortex force in the real ocean (see Section 3 for a detailed discussion).

The VL coordinate used here was originally introduced (in prototype form) by Mellor (2003) and forms the basis of Mellor (2003, 2005). The analysis of Mellor (2003) uses thickness-weighted averaging in a coordinate system that is effectively the same as our VL coordinate system. This is the reason that Mellor (2003) successfully reproduces the vertically integrated results of LHS64 without the need to introduce a delta function at the sea surface in the expression for the depthdependent radiation stress. As noted in section $5 b$, the treatment adopted by Mellor (2003) is nevertheless not ideally suited to the situation of a sloping bottom. We suggest that the analysis in Mellor (2003) is correct as long as the slope is at most $O\left(\alpha^{2}\right)$. For the treatment of a sloping bottom of $O(\alpha)$ we recommend the use of our 
Eq. (32) for which the velocity variable inside the time derivative is the Eulerian mean (not the Lagrangian mean) velocity, which is as in the three-dimensional Lagrangian mean framework of AM78.

Of particular note is the absence in our analysis of a delta function term at the height of the mean sea surface in the expression for the depth-dependent radiation stress, as has been advocated by Mellor (2008, 2011a,b). This is despite the close similarly between the approach adopted here and that advocated by Mellor $(2003,2005)$. We suggest that the reason for this difference is that Mellor (2008, 2011a,b) uses (Eulerian) averaging at fixed height to average the vertical momentum equation and part of the horizontal momentum equation (see section $5 \mathrm{c}$ ). Vertically integrating these equations does not give the same result as averaging the vertically integrated equations, as in LHS64. The lack of interchangability hinges on the undulating free surface, the treatment of which forced Mellor to introduce the delta function into the radiation stress. By contrast, the average of the vertical integral of any variable in Eulerian coordinates is the same as the vertical integral of the TWM of that same variable in the VL coordinates [i.e. $\frac{T}{\int_{-h}^{\eta} A d z^{c}}=\int_{-h}^{\bar{\eta}} \hat{A} d z$ in (41)]. It is interesting that (D2) in appendix D turns out to be a cornerstone in both surface wave literature and mesoscale eddy literature in oceanography.

Acknowledgments. Comments from two anonymous reviewers are gratefully acknowledged. HA thanks Hitoshi Tamura for helpful discussions. RJG is grateful for continuing support from GEOMAR.

\section{APPENDIX A}

\section{The Nondimensionalized Equations of Motion and the Transformation to the Vertically Lagrangian Coordinate System}

The equation system of AG12 and the nondimensionalization are briefly explained here.

\section{a. Eulerian coordinates}

We consider incompressible inviscid water of constant, uniform density in a nonrotating frame. Let an arbitrary variable with an associated physical dimension be expressed by Á, and Eulerian-Cartesian coordinates be labeled by the set of independent variables, $\left(\hat{x}^{c}, \hat{y}^{c}, \hat{z}^{c}, \hat{t}^{c}\right)$, where $\hat{x}^{c}, \hat{y}^{c}$ are horizontal coordinates and $z^{c}$ (the geopotential height) increases vertically upward and $(\dot{u}, \dot{v}, \dot{w})$ are the corresponding three-dimensional components of velocity. The continuity, horizontal and vertical momentum equations then take the form,

$$
\begin{aligned}
& \dot{\nabla}^{c} \cdot \dot{\mathbf{V}}+\dot{w}_{\dot{Z}^{c}}=0, \\
& \dot{\rho}\left(\partial_{\dot{t}^{c}}+\dot{\mathbf{V}} \cdot \dot{\nabla}^{c}+\dot{w} \partial_{\dot{z}^{c}}\right) \dot{\mathbf{V}}=-\dot{\boldsymbol{V}}^{c} p^{\prime}-\underbrace{\dot{\boldsymbol{V}}^{c}\left[\dot{\rho} g\left(\dot{\eta}-\dot{z}^{c}\right)\right]}_{\dot{\rho} g \dot{\boldsymbol{V}}^{c} \dot{\eta}}, \\
& \dot{\rho}\left(\partial_{\dot{t}^{c}}+\dot{\mathbf{V}} \cdot \dot{\nabla}^{c}+\dot{w} \partial_{\dot{z}^{c}}\right) \dot{w}=-\dot{p}_{\dot{z}^{c}},
\end{aligned}
$$

where $\mathbf{\mathbf { V }} \equiv(\dot{u}, \hat{v})$ is the horizontal velocity vector, $\dot{\nabla}^{c}=\left(\partial_{\dot{x}^{c}}, \partial_{\dot{y}^{c}}\right)$ is the horizontal gradient operator, and $\dot{\rho} g\left(\dot{\eta}-\dot{z}^{c}\right)$ is hydrostatic pressure which vanishes at the sea surface where $\dot{z}^{c}=\dot{\eta}$ with $\dot{g}$ being the acceleration due to gravity. Use of the hydrostatic pressure has led to no gravitational acceleration term appearing in (A1c). The quantity $\dot{p}$ is the sum of oceanic nonhydrostatic pressure and atmospheric sea surface pressure.

We nondimensionalize (A1a)-(A1c) using a length scale $\dot{\Delta}$ and associated scales for the other variables,

$$
\begin{aligned}
\left(\hat{x}^{c}, \dot{y}^{c}, \dot{z}^{c}, \dot{\eta}\right) & =\dot{\Delta}\left(x^{c}, y^{c}, z^{c}, \eta\right), \quad \dot{t}^{c}=\left(\dot{\Delta} / g^{\prime}\right)^{1 / 2} t^{c} \\
(\dot{u}, \dot{v}, \dot{w}) & =(\dot{g} \dot{\Delta})^{1 / 2}(u, v, w), \quad \dot{p}=(\dot{\rho} g \dot{\Delta}) p
\end{aligned}
$$

where dimensionless variables are expressed without the acute symbol. Substitution of (A2a)-(A2b) to (A1a)(A1c) yields

$$
\begin{gathered}
\nabla^{c} \cdot \mathbf{V}+w_{z^{c}}=0, \\
\left(\partial_{t^{c}}+\mathbf{V} \cdot \nabla^{c}+w \partial_{z^{c}}\right) \mathbf{V}=-\nabla^{c} p-\underbrace{\nabla^{c}\left[\left(\eta-z^{c}\right)\right]}_{\nabla^{c} \eta}, \\
\left(\partial_{t^{c}}+\mathbf{V} \cdot \nabla^{c}+w \partial_{z^{c}}\right) w=-p_{z^{c}} .
\end{gathered}
$$

This nondimensionalization is for the purpose of convenience and does not restrict the dynamics of the fluid motions.

\section{b. Vertically Lagrangian (VL) coordinates}

AM78 (see their p. 612) have developed a hybrid Lagrangian-Eulerian coordinate system. The idea is to choose a coordinate system that follows the high-frequency fluid motion (i.e., waves), as in Lagrangian coordinates, but is such that the equations for the low-frequency fluid motion (i.e., circulation) appear as in Eulerian coordinates. The hybrid coordinate system of AM78 has been adopted only in the vertical direction in AG12, and is called the VL coordinate system. In the horizontal the 
standard Eulerian coordinates are retained. ${ }^{\mathrm{A} 1}$ Prototypes of the VL coordinate system (i.e., one-dimensional analog of AM78) have been developed by Iwasaki (2001) and Jacobson and Aiki (2006) to describe large-scale hydrostatic circulation in the atmosphere and ocean, respectively.

The VL coordinates are labeled by the set of independent variables $(x, y, z, t)$. The horizontal coordinates $x$ and $y$ are the same as the standard Eulerian-Cartesian coordinates. The transformation between the Eulerian coordinates and the VL coordinates may be written

$$
x^{c}=x, \quad y^{c}=y, \quad z^{c}=z^{c}(x, y, z, t), \quad t^{c}=t,
$$

with the inverse transformation given by

$$
x=x^{c}, \quad y=y^{c}, \quad z=z\left(x^{c}, y^{c}, z^{c}, t^{c}\right), \quad t=t^{c} .
$$

The value of the vertical coordinate $z$ attached to a particular fluid particle at the horizontal location $\left(x^{c}, y^{c}\right)$ at time $t^{c}$ is assigned as follows. First, we let $z^{L}$ be the (Lagrangian) low-pass filtered height of that same fluid particle centered around time $t^{c}$. Then we form the material surface that consists of all fluid particles with this same low-pass filtered height, $z^{L}$, centered around time $t^{c}$. We then define $z$ to be the (Eulerian) low-pass filtered height of this material surface at the location $\left(x^{c}, y^{c}\right)$ and again centered around the time $t^{c}$. It follows immediately that

$$
z \equiv \overline{z^{c}}
$$

where the overbar indicates a low-pass temporal filter carried out in the VL coordinates. To proceed with the mathematical development, we note that spatial derivatives in the $\mathrm{VL}$ coordinates are given by

$$
\left(\begin{array}{c}
\partial_{x} \\
\partial_{y} \\
\partial_{z} \\
\partial_{t}
\end{array}\right)=\left(\begin{array}{llll}
1 & 0 & z_{x}^{c} & 0 \\
0 & 1 & z_{y}^{c} & 0 \\
0 & 0 & z_{z}^{c} & 0 \\
0 & 0 & z_{t}^{c} & 1
\end{array}\right)\left(\begin{array}{c}
\partial_{x^{c}} \\
\partial_{y^{c}} \\
\partial_{z^{c}} \\
\partial_{t^{c}}
\end{array}\right) .
$$

\footnotetext{
A1 The differences between the three-dimensional Lagrangian coordinates of AM78 and the VL coordinates are illustrated in Fig. 2. As the wave propagates rightward, the control cell of the three-dimensional Lagrangian coordinates (blue) rotates clockwise and returns to the original position. The fact that the cell does not drift away (despite of the presence of the Stokes-drift and the Eulerian mean flow) is attributed to the use of the hybrid Lagrangian-Eulerian coordinates in AM78. The control cell of the VL coordinates (red) moves like a piston whose thickness stretches and shrinks. As indicated by the horizontal black lines, the mean vertical position of the control cell in the three-dimensional Lagrangian coordinates is misaligned with that in the VL coordinates (McIntyre 1988).
}

We also note that $z \equiv \overline{z^{c}}$ leads to

$$
\left(\overline{z_{x}^{c}}, \overline{z_{y}^{c}}, \overline{z_{z}^{c}}, \overline{z_{t}^{c}}\right)=(0,0,1,0)
$$

identities that are useful when we average the governing equations. It should also be noted that $z_{z}^{c}$ corresponds to the thickness in isopycnal coordinates (e.g., Andrews 1983; Greatbatch 1998; Greatbatch and McDougall 2003; Aiki and Richards 2008; Tsujino et al. 2010; Young 2012).

Equation (A7) may be used to write the governing equations (A3a)-(A3c) in terms of the VL coordinates to yield (1a)-(1d).

\section{APPENDIX B}

\section{Second-Order Waves Associated with Nonlinear Terms}

Substitution of the $O(\alpha)$ solution $(17 \mathrm{c})-(17 \mathrm{f})$ to the $O\left(\alpha^{2}\right)$ Eqs. (23a)-(23d) yields

$$
\begin{array}{r}
z_{2 z \tau}^{\prime \prime \prime}+\dot{\mathbf{\nabla}} \cdot \mathbf{V}_{2}^{\prime \prime \prime}+\left(\phi_{1}^{\prime \prime \prime} \phi_{1}^{\prime \prime \prime}\right)_{\theta} \sigma \kappa^{4}=0, \\
z_{2 \tau}^{\prime \prime \prime}+\phi_{1}^{\prime \prime \prime} \phi_{1 \theta z}^{\prime \prime \prime} \sigma \kappa^{2}=w_{2}^{\prime \prime \prime}, \\
\mathbf{V}_{2 \tau}^{\prime \prime \prime}+\phi_{1}^{\prime \prime \prime} \phi_{1 \theta}^{\prime \prime \prime} \sigma^{2} \kappa^{2} \dot{\nabla} \theta=-\dot{\nabla}\left(p_{2}^{\prime \prime \prime}+\eta_{2}^{\prime \prime \prime}\right)+\phi_{1 z}^{\prime \prime \prime} \phi_{1 z \theta}^{\prime \prime \prime} \sigma^{2} \dot{\nabla} \theta \\
w_{2 \tau}^{\prime \prime \prime}+\phi_{1}^{\prime \prime \prime} \phi_{1 z}^{\prime \prime \prime} \sigma^{2} \kappa^{2}=-p_{2 z}^{\prime \prime \prime}+\phi_{1 z}^{\prime \prime \prime} \phi_{1}^{\prime \prime \prime} \sigma^{2} \kappa^{2},
\end{array}
$$

where $\dot{\nabla} \phi_{1}^{\prime \prime \prime}=\phi_{1 \theta}^{\prime \prime \prime} \dot{\nabla} \theta, \quad \phi_{1 \tau}^{\prime \prime \prime}=-\phi_{1 \theta}^{\prime \prime \prime} \sigma, \quad \phi_{1 \theta \theta}^{\prime \prime \prime}=-\phi_{1}^{\prime \prime \prime}, \quad$ and $\phi_{1 z z}^{\prime \prime \prime}=\kappa^{2} \phi_{1}^{\prime \prime \prime}$ have been used.

Substitution of (17b) to (B1a)-(B1d) yields

$$
\begin{aligned}
\dot{\nabla} \cdot \mathbf{V}_{2}^{\prime \prime \prime}+w_{2 z}^{\prime \prime \prime} & =-\sin 2 \theta \frac{1+\cosh 2 \kappa(z+h)}{\sinh ^{2} \kappa H} \mathcal{A}^{2} \sigma \kappa^{2}, \\
z_{2 \tau}^{\prime \prime \prime} & =w_{2}^{\prime \prime \prime}+\sin 2 \theta \frac{\sinh 2 \kappa(z+h)}{4 \sinh ^{2} \kappa H} \mathcal{A}^{2} \sigma \kappa, \\
\mathbf{V}_{2 \tau}^{\prime \prime \prime} & =-\dot{\nabla}\left(p_{2}^{\prime \prime \prime}+\eta_{2}^{\prime \prime \prime}\right)+\sin 2 \theta \frac{1}{\sinh ^{2} \kappa H} \mathcal{A}^{2} \sigma^{2} \dot{\nabla} \theta \\
w_{2 \tau}^{\prime \prime \prime} & =-p_{2 z}^{\prime \prime \prime},
\end{aligned}
$$

where $H \equiv \bar{\eta}+h$. Using a bottom boundary condition of $\left.w_{2}^{\prime \prime \prime}\right|_{z=-h}=0$, we solve (B2a)-(B2d) and obtain, 


$$
\begin{gathered}
\mathbf{V}_{2}^{\prime \prime \prime}=\cos 2 \theta \frac{2-2 \kappa(z+h) \sinh 2 \kappa(z+h)+2 \mathcal{B} \cosh 2 \kappa(z+h)}{4 \sinh ^{2} \kappa H} \mathcal{A}^{2} \sigma \dot{\mathbf{\nabla}} \theta, \\
w_{2}^{\prime \prime \prime}=\sin 2 \theta \frac{-\sinh 2 \kappa(z+h)-2 \kappa(z+h) \cosh 2 \kappa(z+h)+2 \mathcal{B} \sinh 2 \kappa(z+h)}{4 \sinh ^{2} \kappa H} \mathcal{A}^{2} \sigma \kappa, \\
z_{2}^{\prime \prime \prime}=\cos 2 \theta \frac{-\kappa(z+h) \cosh 2 \kappa(z+h)+\mathcal{B} \sinh 2 \kappa(z+h)}{4 \sinh ^{2} \kappa H} \mathcal{A}^{2} \kappa, \\
p_{2}^{\prime \prime \prime}+\eta_{2}^{\prime \prime \prime}=\cos 2 \theta \frac{-2 \kappa(z+h) \sinh 2 \kappa(z+h)+2 \mathcal{B} \cosh 2 \kappa(z+h)}{4 \sinh ^{2} \kappa H} \mathcal{A}^{2} \sigma^{2},
\end{gathered}
$$

where $\left.\eta_{2}^{\prime \prime \prime} \equiv z_{2}^{\prime \prime \prime}\right|_{z=0}$ is understood. Parameter $\mathcal{B}$ may be determined from a surface boundary condition $\left.p_{2}^{\prime \prime \prime}\right|_{z=0}=0$ to yield

$$
\begin{aligned}
\mathcal{B} & =\frac{2 \sigma^{2} \sinh 2 \kappa H-\kappa \cosh 2 \kappa H}{2 \sigma^{2} \cosh 2 \kappa H-\kappa \sinh 2 \kappa H} \kappa H \\
& =\frac{2(\tanh \kappa H) \sinh 2 \kappa H-\cosh 2 \kappa H}{2(\tanh \kappa H) \cosh 2 \kappa H-\sinh 2 \kappa H} \kappa H \\
& =\frac{\cosh 3 \kappa H-3 \cosh \kappa H}{\sinh 3 \kappa H-3 \sinh \kappa H} \kappa H,
\end{aligned}
$$

where the dispersion relation (17a) has been used.

\section{APPENDIX C \\ Energy Equations}

The depth-dependent wave energy equations in a general form have been derived by AG12. We take the sum of Eqs. (A6a) and (A6b) of AG12 and pick-up $O\left(\alpha^{3}\right)$ terms to yield

$\frac{1}{2}\left(\overline{\left|\mathbf{V}_{1}^{\prime \prime \prime}\right|^{2}+w_{1}^{\prime \prime \prime}}\right)_{T}+\overline{\left[z_{1 T}^{\prime \prime \prime}\left(p_{1}^{\prime \prime \prime}+\eta_{1}^{\prime \prime \prime}\right)\right.}+\overline{z_{1 \tau}^{\prime \prime \prime}\left(p_{2}^{\prime \prime \prime}+\eta_{2}^{\prime \prime \prime}\right)}+\overline{\left.z_{2 \tau}^{\prime \prime \prime}\left(p_{1}^{\prime \prime \prime}+\eta_{1}^{\prime \prime \prime}\right)\right]_{z}}+\bar{\nabla} \cdot \overline{\left[\mathbf{V}_{1}^{\prime \prime \prime}\left(p_{1}^{\prime \prime \prime}+\eta_{1}^{\prime \prime \prime}\right)\right]}+\overline{\left[w_{2}^{* \prime \prime \prime}\left(p_{1}^{\prime \prime \prime}+\eta_{1}^{\prime \prime \prime}\right)\right]_{z}}=0$

which has some similarity to the depth-dependent radiation stress term based on (10a)-(10b),

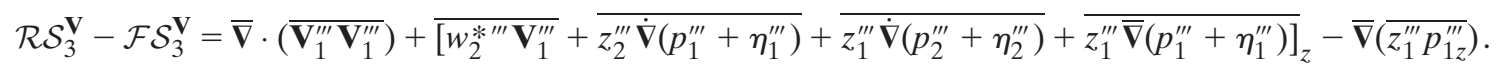

It is left for a future study to rederive (31) using the set of (C1) and (C2) as well as the wave crest equation in order to see how to extend the approach of Garrett (1976) and Smith (2006) to the depth-dependent problem.

\section{APPENDIX D}

\section{Approximated Expressions for the Eulerian Mean}

Using a Taylor expansion in the vertical direction, we express the Eulerian mean of an arbitrary quantity $A$ (averaged in the Eulerian-Cartesian coordinates) in terms of quantities averaged in the $\mathrm{VL}$ coordinates,

$$
\begin{aligned}
\bar{A}^{c} & =\bar{A}+\overline{\left(-z^{\prime \prime \prime}\right)\left(A_{z^{c}}\right)}+\overline{\left(-z^{\prime \prime \prime}\right)^{2}\left(A_{z^{c} z^{c}}\right)} / 2+\cdots \\
& =\bar{A}-\overline{z^{\prime \prime \prime}\left(A_{z} / z_{z}^{c}\right)}+\overline{z^{\prime \prime \prime} 2\left[\left(A_{z} / z_{z}^{c}\right)_{z} / z_{z}^{c}\right]} / 2+\cdots \\
& \left.\simeq \bar{A}-\overline{z^{\prime \prime \prime}\left[\left(\bar{A}_{z}+A_{z}^{\prime \prime \prime}\right)\left(1-z_{z}^{\prime \prime \prime}\right)\right]}+\overline{\left(z^{\prime \prime \prime}\right.} / 2\right) \bar{A}_{z z}+\cdots \\
& \left.\left.=\bar{A}-\overline{z^{\prime \prime \prime} A_{z}^{\prime \prime \prime}}+\overline{\left(z^{\prime \prime \prime}\right.} / 2\right)_{z} \bar{A}_{z}+\overline{\left(z^{\prime \prime \prime}\right.} / 2\right) \bar{A}_{z z}+\cdots \\
& \left.=\bar{A}-\overline{z^{\prime \prime \prime} A_{z}^{\prime \prime \prime}}+\left[\overline{\left(z^{\prime \prime \prime}\right.} / 2\right) \bar{A}_{z}\right]_{z}+\cdots
\end{aligned}
$$

Substitution of $A=p$ to (D1) and the use of (12c) yields the first line of (33). Using (D1) we express the TWM quantity as

$$
\begin{aligned}
\hat{A} & \equiv \overline{z_{z}^{c} A}=\bar{A}+\overline{z_{z}^{\prime \prime \prime} A^{\prime \prime \prime}} \\
& \left.=\bar{A}^{c}+\left(\overline{z^{\prime \prime \prime} A^{\prime \prime \prime}}\right)_{z}-\left[\overline{\left(z^{\prime \prime \prime}\right.} / 2\right) \bar{A}_{z}\right]_{z}-\cdots \\
& =\bar{A}^{c}+\left[\overline{z^{\prime \prime \prime} A^{\prime \prime \prime}}-\left(\overline{z^{\prime \prime \prime}} / 2\right) \bar{A}_{z}\right]_{z}-\cdots
\end{aligned}
$$


which is a cornerstone for computing the difference of the depth integral of the TWM and Eulerian mean quantities. $^{\text {D1 }}$

\section{REFERENCES}

Aiki, H., and T. Yamagata, 2006: Energetics of the layer-thickness form drag based on an integral identity. Ocean Sci., 2, 161-171.

— role of layer-thickness form drag. J. Phys. Oceanogr., 38, 18451869.

— , and R. J. Greatbatch, 2012: Thickness-weighted mean theory for the effect of surface gravity waves on mean flows in the upper ocean. J. Phys. Oceanogr., 42, 725-747.

Andrews, D. G., 1983: A finite-amplitude Eliassen-Palm theorem in isentropic coordinates. J. Atmos. Sci., 40, 1877-1883.

— , and M. E. McIntyre, 1978: An exact theory of nonlinear waves on a Lagrangian-mean flow. J. Fluid Mech., 89, 609-646.

Ardhuin, F., A. D. Jenkins, and K. A. Belibassakis, 2008a: Comments on "The three-dimensional current and surface wave equations" by George Mellor. J. Phys. Oceanogr., 38, 1340-1350.

—, N. Rascle, and K. Belibassakis, 2008b: Explicit waveaveraged primitive equations using a generalized Lagrangian mean. Ocean Modell., 20, 35-60.

Bennis, A.-C., and F. Ardhuin, 2011: Comments on "The depthdependent current and wave interaction equations: A revision." J. Phys. Oceanogr., 41, 2008-2012.

Broström, G., K. H. Christensen, and J. E. H. Weber, 2008: A quasi-Eulerian, quasi-Lagrangian view of surface-waveinduced flow in the ocean. J. Phys. Oceanogr., 38, 1122-1130.

Chu, V. H., and C. C. Mei, 1970: On slowly varying Stokes waves. J. Fluid Mech., 41, 873-887.

Craik, A. D. D., 1985: Wave Interactions and Fluid Flows. Cambridge University Press, $322 \mathrm{pp}$.

_ culations. J. Fluid Mech., 73, 401-426.

Fan, Y., I. Ginis, and T. Hara, 2010: Momentum flux budget across the air-sea interface under uniform and tropical cyclone winds. J. Phys. Oceanogr., 40, 2221-2242.

Garrett, C., 1976: Generation of Langmuir circulations by surface waves - A feedback mechanism. J. Mar. Res., 34, 117-130.

Greatbatch, R. J., 1998: Exploring the relationship between eddyinduced transport velocity, vertical momentum transfer, and the isopycnal flux of potential vorticity. J. Phys. Oceanogr., 28, $422-432$.

${ }^{\mathrm{D} 1}$ The vertical integral of the second term on the last line of (D2) vanishes if the sea surface is assumed to be rigid as in theoretical studies for mesoscale eddies (i.e., $z^{\prime \prime \prime}=0$ at both the top and bottom of the ocean), allowing McDougall and McIntosh (2001) to show that the quasi-Stokes velocity $\mathbf{V}^{q s} \equiv \hat{\mathbf{V}}-\overline{\mathbf{V}}^{c}$ associated with mesoscale eddies in a stratified fluid has no depth-averaged component. Substitution of $A^{\prime \prime \prime} \simeq A^{\prime}+z^{\prime \prime \prime} \bar{A}_{z^{c}}^{c}$ (where $A^{\prime}$ is the deviation from the Eulerian mean, Table 1) to (D2) yields, $\hat{A} \simeq \bar{A}^{c}+\left[\overline{z^{\prime \prime \prime} A^{\prime}}+\right.$ $\left.\left(\overline{z^{\prime \prime \prime}}\right) \bar{A}_{z^{c}}^{c}-\left(\overline{z^{\prime \prime \prime}} / 2\right) \bar{A}_{z}\right]_{z} \simeq \bar{A}^{c}+\left[\overline{z^{\prime \prime \prime} A^{\prime}}+\left(\overline{z^{\prime \prime \prime 2}} / 2\right) \bar{A}_{z^{c}}^{c}\right]_{z}$, which is identical to Eq. (A5) of McDougall and McIntosh (2001) (they used an additional approximation $z^{\prime \prime \prime} \simeq-\gamma^{\prime} / \bar{\gamma}_{z^{c}}^{c}$ where $\gamma$ is density, which is specialized to depths away from the top and bottom boundaries). The above argument has led to the pile-up rule of Aiki and Yamagata (2006).
— , and T. J. McDougall, 2003: The non-Boussinesq temporal residual mean. J. Phys. Oceanogr., 33, 1231-1239.

Iwasaki, T., 2001: Atmospheric energy cycle viewed from wavemean-flow interaction and Lagrangian mean circulation. J. Atmos. Sci., 58, 3036-3052.

Jacobson, T., and H. Aiki, 2006: An exact energy for TRM theory. J. Phys. Oceanogr., 36, 558-564.

Lane, E. M., J. M. Restrepo, and J. C. McWilliams, 2007: Wavecurrent interaction: A comparison of radiation-stress and vortex-force representations. J. Phys. Oceanogr., 37, 11221141.

Lentz, S. J., and M. R. Fewings, 2012: The wind- and wave-driven inner-shelf circulation. Annu. Rev. Mar. Sci., 4, 317-343.

Longuet-Higgins, M. S., and R. W. Stewart, 1964: Radiation stress in water waves: A physical discussion with applications. DeepSea Res., 11, 529-562.

McDougall, T. J., and P. C. McIntosh, 2001: The temporal-residualmean velocity. Part II: Isopycnal interpretation and the tracer and momentum equations. J. Phys. Oceanogr., 31, 12221246.

McIntyre, M. E., 1988: A note on the divergence effect and the Lagrangian-mean surface elevation in periodic water waves. J. Fluid Mech., 189, 235-242.

McWilliams, J. C., J. M. Restrepo, and E. M. Lane, 2004: An asymptotic theory for the interaction of waves and currents in coastal waters. J. Fluid Mech., 511, 135-178.

Mellor, G., 2003: The three-dimensional current and surface wave equations. J. Phys. Oceanogr., 33, 1978-1989.

- 2005: Some consequences of the three-dimensional current and surface wave equations. J. Phys. Oceanogr., 35, 22912298.

- 2008: The depth-dependent current and wave interaction equations: a revision. J. Phys. Oceanogr., 38, 2587-2596.

, 2011a: Wave radiation stress. Ocean Dyn., 61, 563-568.

, 2011b: Corrigendum. J. Phys. Oceanogr., 41, 1417-1418.

Smith, J. A., 2006: Wave-current interactions in finite depth. J. Phys. Oceanogr., 36, 1403-1419.

Tsujino, H., S. Nishikawa, K. Sakamoto, H. Nakano, and H. Ishizaki, 2010: Mesoscale eddy statistics and implications for parameterization refinements from a diagnosis of a high resolution model of the North Pacific. Ocean Modell., 33, 205-223.

Uchiyama, Y., J. C. McWilliams, and J. M. Restrepo, 2009: Wavecurrent interaction in nearshore shear instability analyzed with a vortex force formalism. J. Geophys. Res., 114, C06021, doi:10.1029/2008JC005135.

- — - and A. F. Shchepetkin, 2010: Wave-current interaction in an oceanic circulation model with a vortex-force formalism: Application to the surf zone. Ocean Modell., 34, 16-35.

Young, W. R., 2012: An exact thickness-weighted average formulation of the Boussinesq equations. J. Phys. Oceanogr., 42, 692-707.

Zou, Q., A. E. Hay, and A. J. Bowen, 2003: Vertical structure of surface gravity waves propagating over a sloping seabed: Theory and field measurements. J. Geophys. Res., 108, C83265, doi:10.1029/2002JC001432. 